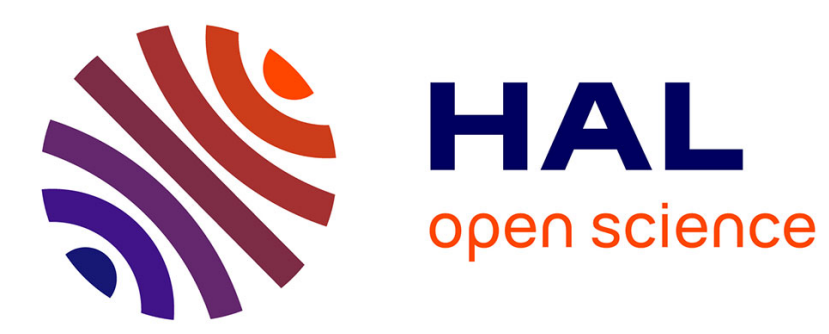

\title{
Three-dimensional modeling of elastic guided waves excited by arbitrary sources in viscoelastic multilayered plates
}

Fabien Treyssede

\section{- To cite this version:}

Fabien Treyssede. Three-dimensional modeling of elastic guided waves excited by arbitrary sources in viscoelastic multilayered plates. Wave Motion, 2015, 52, pp.33-53. 10.1016/j.wavemoti.2014.08.007 . hal-01346099

\section{HAL Id: hal-01346099 \\ https://hal.science/hal-01346099}

Submitted on 18 Jul 2016

HAL is a multi-disciplinary open access archive for the deposit and dissemination of scientific research documents, whether they are published or not. The documents may come from teaching and research institutions in France or abroad, or from public or private research centers.
L'archive ouverte pluridisciplinaire HAL, est destinée au dépôt et à la diffusion de documents scientifiques de niveau recherche, publiés ou non, émanant des établissements d'enseignement et de recherche français ou étrangers, des laboratoires publics ou privés. 


\title{
Three-dimensional modeling of elastic guided waves excited by arbitrary sources in viscoelastic multilayered plates
}

\author{
Fabien Treyssède ${ }^{\mathrm{a}, *}$, \\ ${ }^{a}$ LUNAM Université, IFSTTAR, Route de Bouaye, 44344 Bouguenais, France
}

\begin{abstract}
This paper provides a modal solution for the three-dimensional modeling of Lamb and SH waves excited by sources of arbitrary shape. This solution is applicable to elastic and viscoelastic plates, in the far-field as well as in the nearfield regions, under the assumption of transverse isotropy about the thickness direction. The theoretical developments are conducted based on a semi-analytical finite element formulation. This formulation yields a one-dimensional modal problem, fast from a computational point of view, and allows to readily handle heterogeneous materials having depth-varying properties (multilayered, piecewise or continuously varying, functionally graded). The modal solution is shown to be expressed in terms of Hankel functions of multiple order thanks to a proper application of inverse transforms and Cauchy residue calculus. The link between the proposed formulation and a fully analytical approach is discussed. The solution of this paper is then successfully compared to literature results and degenerates to the point source case. Formula are presented to calculate point source excitabilities from lines sources. These formula remain valid for non-propagating modes, viscoelastic materials and account for the near-field contribution. Finally, the example of a viscoelastic bilayer waveguide excited by a rectangular source is considered in order to check the theoretical results.
\end{abstract}

Keywords: waveguide, plate, excitation, three-dimensional modeling, viscoelastic, excitability

\section{Introduction}

Lamb waves are of great interest for the non-destructive testing and the health monitoring of plate-like structures. Such waves are dispersive and multimodal, which complicates their practical use. Dispersion curves of phase and group velocities as functions of frequency are useful to identify modes that propagate in a frequency range with low dispersion and low attenuation [1]. These curves represent modal properties obtained regardless excitation. For a practical inspection system, it is also essential to determine and control the amplitudes of each guided modes excited by a given source. This information typically allows to optimize the type and location of sensors to be used.

The 2D modeling of Lamb wave excitation and propagation has been extensively studied. Two approaches can mainly be distinguished in order to calculate the response of waveguides under excitation. A first approach consists in using integral transform techniques [2, 3, 4, 5, 6, 7]. With this method, the response is obtained by contour integration in the complex plane and residue calculus, or numerical integration, of the analytical solution expressed in the wavenumber domain.

An alternative approach is based on modal analysis, which consists in expanding the excited field as a sum of guided modes $[8,9,10]$. The contribution of each mode is obtained from an orthogonality relation between eigenmodes. This second method appears to be more suitable to achieve a better interpretation and optimization of signals, as it directly provides the contribution of each mode as a function of the excitation. Additionally, this method has allowed to introduce the useful concept of modal excitability $[11,12,13,14]$. For a given frequency, the excitability

\footnotetext{
${ }^{*}$ Corresponding author

Email address: fabien.treyssede@ifsttar.fr (Fabien Treyssède )
} 
of a particular mode can be defined as the ratio of the displacement of that mode to a point force applied in a given direction.

However, the plane-strain assumption inherently used for 2D modeling implies that guided waves are not geometrically attenuated as they propagate and that any source actually extends infinitely along the out-of-plane direction (a 2D point force hence corresponds to an out-of-plane line source). A 3D formulation is required in order to account for a finite length excitation, typically generated by transducers.

The 3D modeling of Lamb wave propagation generated by finite sources is more complex. Based on contour integration and residue calculus, point source solutions can be found for isotropic plates [15, 16, 17, 18, 19]. Mainly based on numerical integration, further works can be found for quasi-isotropic [20], multilayered isotropic [21] or anisotropic $[22,23,24]$ plates. However, numerical integration techniques usually require a large amount of computation time to evaluate the double integral of the spatial inverse Fourier transform, which has extremely irregular integrands $[22,23]$.

With modal techniques, a direct way of expressing 3D wave fields in terms of modal expansions has been formulated by Achenbach [9] for an isotropic plate. This novel approach uses reciprocal identities as well as the concept of carrier waves [25] and leads to analytical solutions both for normal and tangential point loads [26]. The advantage of a fully modal technique is to replace the double integral of the inverse Fourier transform with a modal expansion, which is much more efficient from a computational point of view. Based on the work of Achenbach, Wilcox [27] has derived asymptotic far-field 3D modal excitabilities. Under the assumption that no material damping is present, Velichko and Wilcox [13] have further extended these results to generally anisotropic multilayered media. Based on Refs. [2, 18], Moulin et al. [28] have proposed far-field modal solutions for an isotropic plate subjected to a normal surface load of rectangular shape. More generally, problems involving arbitrary sources can be treated by two-dimensional convolution of the point source solution, but this can be costly from a computational point of view. Further improvements are possible.

The purpose of this paper is to provide a 3D modal solution for Lamb and SH waves generalized to sources of arbitrary shape. This solution is restricted to transversely isotropic problems with symmetry axis normal to the plate surface (quasi-isotropy), so that the modal features of the plate do not depend on the propagation angle. This key property allows to achieve fully modal solutions (without integral), expanded as double sums over normal modes and Fourier coefficients of the source. This paper generalizes previous 3D modal solutions usually restricted to point sources $[26,27,13]$. Furthermore, the proposed modal solution is shown to be applicable to viscoelastic solids as well as in the near field region. It should be mentioned that, while the integral transform approach still applies with complex poles, and thereby to lossy waveguides [29, 24, 30], the validity of modal techniques with complex modes might be unclear. Complex modes typically occur with viscoelastic materials or in near-field calculations, involving evanescent or inhomogeneous modes. In case of 2D plate modeling, it has been recently shown in Ref. [14] that complex modes can be handled with modal expansion techniques thanks to the use of Auld's real biorthogonality relation, instead of Auld's complex relation [8] (the latter only holds for real wavenumbers, i.e. propagating modes in lossless waveguides).

The theoretical developments of this paper are mainly based on a so-called semi-analytical finite element (SAFE) method. Although approximate by nature, such a numerical method allows to readily handle heterogeneous materials having depth-varying properties (multilayered, piecewise or continuously varying). The SAFE modal approach has been essentially developed for studying 3D cylindrical waveguides of arbitrary cross-section, viscoelastic or not (see for instance Refs. [31, 32, 33, 34, 35]), and 2D anisotropic multilayered plates subjected to line loads [36, 37]. For plate structures, the SAFE method restricts the finite element (FE) discretization to only one dimension (along the depth) and is thus fast from a computational point of view. Wave modes can be solved from a matrix eigensystem using standard eigensolvers, which avoids the use of complex root finding algorithms required with fully analytical approaches [38, 39]. A one-dimensional SAFE approach has been specifically proposed by Bai et al. [40] for computing the 3D response of layered isotropic plates. These authors yet restricted their calculation to Green's solution (point source). Besides, the solution was not expressed in terms of Hankel functions, which may limit its practical use. In the present paper, the source is of arbitrary shape and the wavenumber domain of integration is chosen differently, leading to Hankel type solutions.

This paper is organized as follows. Section 2 describes the SAFE formulation required for the 3D modeling of elastic waves. In this formulation, the displacement field retains its three components. Section 3 gives the modal solution. The response is first derived in the wavenumber domain. The response in the space domain is then obtained 
from the application of Cauchy residue theorem. It is shown that the solution is a double sum on Lamb modes and on the Fourier coefficients of the excitation, involving Hankel functions of multiple order. The link between the SAFE solution and a fully analytical approach is also established. In Sec. 4, the modal solution is validated with literature results. Formula are presented to calculate point source excitabilities from lines sources. A discussion on orthogonality relations is provided to highlight the closed link existing between the SAFE biorthogonality relation, the real biorthogonality relation of Auld [8] and the relation of Fraser [41]. Section 5 finally shows some examples of SAFE calculations for a rectangular source exciting a viscoelastic bilayer plate. Results are compared with those obtained from a double convolution of the point source solution.

One points out that plate problems of more general anisotropy, including transverse isotropy with symmetry axis parallel to the plate surface, cannot be considered by the proposed approach. In such problems, modal properties depend on the propagation angle and, therefore, must be computed as a function of that angle. As shown in Ref. [42] for point sources, the response can then be obtained as a sum over normal modes by application of Cauchy residue theorem and a finite integral over a full circumferential sweep, which has to be approximated by numerical quadrature. If attention is restricted to the far-field region in undamped media, this circumferential integral can be avoided thanks to the stationary phase method $[13,43]$ (but the computation of modal properties as a function of the propagation angle is still necessary in order to calculate the stationary point).

\section{Equations of motion}

\subsection{Change of basis}

One assumes a linear elastic material, small strains and displacements with a time harmonic $e^{-i \omega t}$ dependence. In a fixed reference Cartesian coordinate system $(x, y, z)$, the elastodynamic equilibrium equation is:

$$
\nabla \cdot \sigma+\rho \omega^{2} \mathbf{u}=-\mathbf{f}
$$

with the stress-strain relationship $\sigma=\mathbf{C}: \boldsymbol{\epsilon}$. $\mathbf{u}, \rho$ and $\mathbf{f}$ denote the displacement, mass density and volume sources respectively. Following Refs. [40,42], a coordinate transformation must first be applied to rewrite the equilibrium equations in the direction of the traveling waves.

A two-dimensional Fourier transform is applied along the directions $x$ and $y$, defined for an arbitrary function $f(x, y)$ as:

$$
\tilde{f}\left(k_{x}, k_{y}\right)=\int_{-\infty}^{+\infty} \int_{-\infty}^{+\infty} f(x, y) e^{-i\left(k_{x} x+k_{y} y\right)} d x d y
$$

$k_{x}$ and $k_{y}$ are the wavenumbers in the $x$ and $y$ directions, respectively. In this paper, the tilde is used to denote twodimensional Fourier transforms. With the assumption that material properties remain constant along $x$ and $y$, spatial derivatives in Eq. (1) are thus transformed as follows:

$$
\frac{\partial}{\partial x} \longrightarrow i k_{x}, \quad \frac{\partial}{\partial y} \longrightarrow i k_{y}
$$

Note that the variation of properties is allowed in the $z$ direction (depth, see Fig. 1).

Let us apply a polar change of variable from $\left(k_{x}, k_{y}\right)$ to $(k, \phi)$, with:

$$
k_{x}=k \cos \phi, \quad k_{y}=k \sin \phi
$$

A new coordinate system $\left(x^{\prime}, y^{\prime}, z\right)$ is defined, $\left(x^{\prime}, y^{\prime}\right)$ being oriented at angle $\phi$ from $(x, y)$ (see Fig. 1). $k$ hence represents the wavenumber in the direction $x^{\prime}$. Let us define the following matrix:

$$
\mathbf{J}=\left[\begin{array}{ccc}
\cos \phi & \sin \phi & 0 \\
-\sin \phi & \cos \phi & 0 \\
0 & 0 & 1
\end{array}\right]
$$

$\mathbf{J}$ is an orthogonal transformation matrix $\left(\mathbf{J}^{-1}=\mathbf{J}^{T}\right)$, representing a rotation of angle $\phi$ around the axis $z$. The coordinate systems $\left(x^{\prime}, y^{\prime}, z\right)$ and $(x, y, z)$ are linked through: $\mathbf{x}^{\prime}=\mathbf{J x}$, where $\mathbf{x}=\left[\begin{array}{lll}x & y & z\end{array}\right]^{T}$ and $\mathbf{x}^{\prime}=\left[\begin{array}{lll}x^{\prime} & y^{\prime} & z\end{array}\right]^{T}$. For spatial derivatives, one has:

$$
\frac{\partial}{\partial \mathbf{x}^{\prime}}=\mathbf{J} \frac{\partial}{\partial \mathbf{x}}
$$




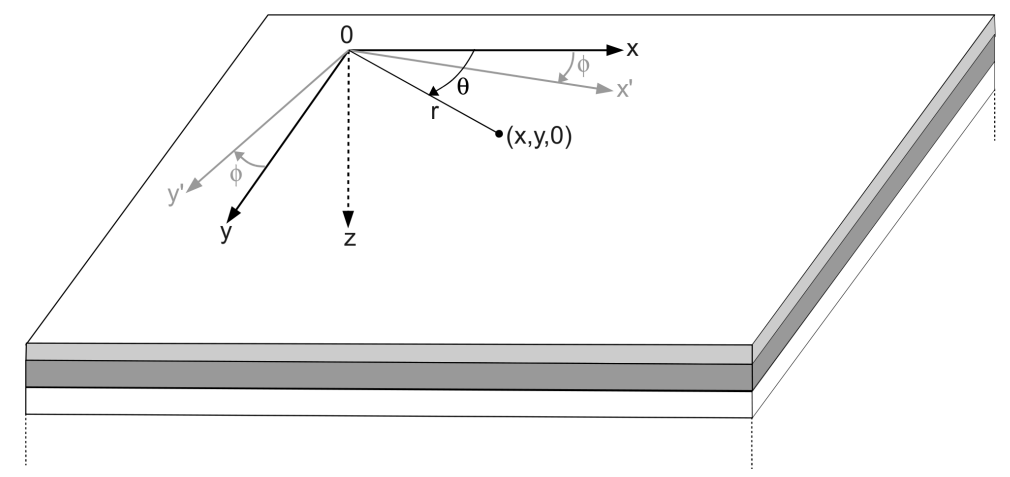

Figure 1: Coordinate systems associated with a plate. $z$ is the depth. $x^{\prime}$ corresponds to the propagation direction in the $(k, \phi)$ space.

Under this transformation, the equilibrium equation (1) becomes:

$$
\nabla^{\prime} \cdot \sigma^{\prime}+\rho \omega^{2} \mathbf{u}^{\prime}=-\mathbf{f}^{\prime}
$$

with $\mathbf{u}^{\prime}=\mathbf{J u}$ and $\mathbf{f}^{\prime}=\mathbf{J f}$. Primes are used for denoting vectors and tensors expressed in the basis $\left(\mathbf{e}_{x^{\prime}}, \mathbf{e}_{y^{\prime}}, \mathbf{e}_{z}\right)$, attached to the $\left(x^{\prime}, y^{\prime}, z\right)$ frame. Materials are assumed transversely isotropic about the thickness direction (that is with symmetry axis normal to the plate surface) so that the elasticity tensor $\mathbf{C}$ remains invariant under the rotation $\mathbf{J}$ $\left(\mathbf{C}=\mathbf{C}^{\prime}\right)$. Hence the stress-strain relationship can be written as:

$$
\sigma^{\prime}=\mathbf{C}: \epsilon^{\prime}
$$

where $\epsilon^{\prime}=\mathbf{J} \epsilon \mathbf{J}^{T}$ and $\sigma^{\prime}=\mathbf{J} \sigma \mathbf{J}^{T}$.

\subsection{SAFE formulation}

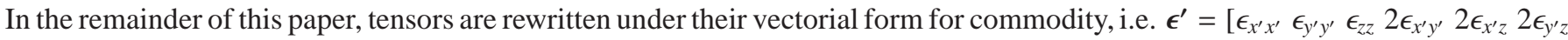
and $\boldsymbol{\sigma}^{\prime}=\left[\begin{array}{llll}\sigma_{x^{\prime} x^{\prime}} & \sigma_{y^{\prime} y^{\prime}} & \sigma_{z z} & \sigma_{x^{\prime} y^{\prime}} \\ \sigma_{x^{\prime} z} & \sigma_{y^{\prime} z}\end{array}\right]^{T}$. One has $\boldsymbol{\sigma}^{\prime}=\mathbf{C} \boldsymbol{\epsilon}^{\prime}$, where the matrix $\mathbf{C}$ of a transverse isotropic material is:

$$
\mathbf{C}=\left[\begin{array}{cccccc}
C_{11} & C_{12} & C_{13} & 0 & 0 & 0 \\
C_{12} & C_{11} & C_{13} & 0 & 0 & 0 \\
C_{13} & C_{13} & C_{33} & 0 & 0 & 0 \\
0 & 0 & 0 & \left(C_{11}-C_{12}\right) / 2 & 0 & 0 \\
0 & 0 & 0 & 0 & C_{55} & 0 \\
0 & 0 & 0 & 0 & 0 & C_{55}
\end{array}\right]
$$

The weak variational form associated with Eq. (7) is:

$$
\int_{V} \delta \boldsymbol{\epsilon}^{\prime T} \mathbf{C} \boldsymbol{\epsilon}^{\prime} d V-\omega^{2} \int_{V} \rho \delta \mathbf{u}^{\prime T} \mathbf{u}^{\prime} d V=\int_{V} \delta \mathbf{u}^{\prime T} \mathbf{f}^{\prime} d V+\int_{S} \delta \mathbf{u}^{\prime T} \mathbf{t}^{\prime} d S
$$

where $\delta$ denotes virtual fields and $t_{i}^{\prime}=\sigma_{i j}^{\prime} n_{j}$ is the traction vector applied on the surface $S$.

Using the property (3) as well as Eq. (4) into Eq. (6), it can be checked that the spatial derivatives along $x^{\prime}$ and $y^{\prime}$ are transformed as:

$$
\frac{\partial}{\partial x^{\prime}} \longrightarrow i k, \quad \frac{\partial}{\partial y^{\prime}} \longrightarrow 0
$$

Accounting for the above property, the deformation vector is transformed into the Fourier domain as:

$$
\tilde{\boldsymbol{\epsilon}}^{\prime}=\left(i k \mathbf{L}_{x^{\prime}}+\mathbf{L}_{z} \frac{\partial}{\partial z}\right) \tilde{\mathbf{u}}^{\prime}
$$


with:

$$
\mathbf{L}_{x^{\prime}}=\left[\begin{array}{ccc}
1 & 0 & 0 \\
0 & 0 & 0 \\
0 & 0 & 0 \\
0 & 1 & 0 \\
0 & 0 & 1 \\
0 & 0 & 0
\end{array}\right], \quad \mathbf{L}_{z}=\left[\begin{array}{lll}
0 & 0 & 0 \\
0 & 0 & 0 \\
0 & 0 & 1 \\
0 & 0 & 0 \\
1 & 0 & 0 \\
0 & 1 & 0
\end{array}\right]
$$

From Eqs. (10) and (12), the two-dimensional Fourier transform of the equilibrium equations written in the $\left(x^{\prime}, y^{\prime}, z\right)$ coordinate system yields the following matrix eigenproblem after FE discretization along $z$ :

$$
\left\{\mathbf{K}_{1}-\omega^{2} \mathbf{M}+i k\left(\mathbf{K}_{2}-\mathbf{K}_{2}^{T}\right)+k^{2} \mathbf{K}_{3}\right\} \tilde{\mathbf{U}}^{\prime}=\tilde{\mathbf{F}}^{\prime}
$$

with the elementary matrices:

$$
\begin{array}{r}
\mathbf{K}_{1}^{e}=\int_{z} \mathbf{N}^{e T} \mathbf{L}_{x^{\prime}}^{T} \mathbf{C} \mathbf{L}_{x^{\prime}} \mathbf{N}^{e} d z, \mathbf{K}_{2}^{e}=\int_{z} \mathbf{N}^{e T} \mathbf{L}_{x^{\prime}}^{T} \mathbf{C} \mathbf{L}_{z} \mathbf{N}_{,}^{e} d z, \\
\mathbf{K}_{3}^{e}=\int_{z} \mathbf{N}_{, z}^{e T} \mathbf{L}_{z}^{T} \mathbf{C L}_{z} \mathbf{N}_{, z}^{e} d z, \mathbf{M}^{e}=\int_{z} \rho \mathbf{N}^{e T} \mathbf{N}^{e} d z
\end{array}
$$

On one element of the cross-section, the displacement is interpolated as $\tilde{\mathbf{u}}^{\prime}=\mathbf{N}^{e} \tilde{\mathbf{U}}^{\prime}$, where $\mathbf{N}^{e}$ is the matrix of nodal interpolating functions on the element.

In Eq. (14), $\tilde{\mathbf{U}}^{\prime}$ is the column vector containing transversal nodal displacements in the wavenumber-frequency domain, i.e. function of $k, \phi$ and $\omega$. $\tilde{\mathbf{F}}^{\prime}$ is the excitation vector in the wavenumber-frequency domain, gathering the contributions of both volume sources $\tilde{\mathbf{f}}^{\prime}$ and surface sources $\tilde{\mathbf{t}}^{\prime}$.

\subsection{Eigenproblem}

Suppressing the excitation vector, Eq. (14) corresponds to a quadratic eigenvalue problem. The eigensolutions $\left(k_{m}, \mathbf{U}_{m}^{\prime}\right)$ represent the wavenumber and the displacement vector associated with the $m$ th guided mode (the tilde on $\mathbf{U}_{m}^{\prime}$ is dropped for conciseness of notations).

If $k_{m}$ is an eigenvalue, then $-k_{m}$ is also an eigenvalue due to the symmetry of $\mathbf{K}_{1}, \mathbf{K}_{3}$ and $\mathbf{M}$. Hence, the eigenproblem has two sets of eigensolutions $\left(k_{m}, \mathbf{U}_{m}^{\prime}\right)$ and $\left(k_{-m}, \mathbf{U}_{-m}^{\prime}\right)$, where $k_{-m}=-k_{m}(m=1, \ldots, M)$, representing $M$ positive-going and $M$ negative-going wave modes. The number of modes $2 M$ is theoretically equal to twice the number of degrees of freedom (dofs) involved in the system (14), but the modal basis is usually truncated in practice.

A fundamental property is that displacement components $\left(\tilde{u}_{x^{\prime}}, \tilde{u}_{z}\right)$ and $\tilde{u}_{y^{\prime}}$ are uncoupled. This could be checked from the particular form of $\mathbf{C}$ in transverse anisotropy, recalled by Eq. (9), together with the expression of deformation in the wavenumber domain in the $\left(x^{\prime}, y^{\prime}, z\right)$ system, given by Eq. (12). As a consequence, the $2 M$ eigensolutions of Eq. (14) divide into two kinds of modes:

- $2 M^{L}$ modes corresponding to plane-strain motion in the $\left(x^{\prime}, z\right)$ plane, such that $\tilde{u}_{y^{\prime}}^{L}=0$ (Lamb modes);

- $2 M^{S H}$ modes corresponding to anti-plane motion along the $y^{\prime}$ direction, such that $\tilde{u}_{x^{\prime}}^{S H}=\tilde{u}_{z}^{S H}=0$ (SH modes),

where $2 M=2 M^{L}+2 M^{S H}$. Obviously, the eigenproblem (14) could be divided into two uncoupled eigenproblem problems (as done in Ref. [40]). Yet in this paper, the global formulation (14) is preferred in order to keep more compact expressions.

\section{Solution}

\subsection{Response in the wavenumber domain}

In order to be solved by standard numerical solvers, quadratic eigenproblems must be transformed into linear eigenproblems [44]. Equation (14) can be recast into the following generalized eigenproblem:

$$
(\mathbf{A}-k \mathbf{B}) \hat{\mathbf{U}}^{\prime}=\hat{\mathbf{F}}^{\prime}
$$


with:

$$
\mathbf{A}=\left[\begin{array}{cc}
\mathbf{0} & \mathbf{K}_{1}-\omega^{2} \mathbf{M} \\
\mathbf{K}_{1}-\omega^{2} \mathbf{M} & i\left(\mathbf{K}_{2}-\mathbf{K}_{2}^{T}\right)
\end{array}\right], \mathbf{B}=\left[\begin{array}{cc}
\mathbf{K}_{1}-\omega^{2} \mathbf{M} & \mathbf{0} \\
\mathbf{0} & -\mathbf{K}_{3}
\end{array}\right]
$$

and:

$$
\hat{\mathbf{U}}^{\prime}=\left\{\begin{array}{c}
\tilde{\mathbf{U}}^{\prime} \\
k \tilde{\mathbf{U}}^{\prime}
\end{array}\right\}, \hat{\mathbf{F}}^{\prime}=\left\{\begin{array}{c}
\mathbf{0} \\
\tilde{\mathbf{F}}^{\prime}
\end{array}\right\}
$$

The solution to Eq. (16), which is the forced response under the excitation $\hat{\mathbf{F}}^{\prime}$, is now expanded as a sum of guided modes: $\hat{\mathbf{U}}^{\prime}=\sum_{m=1}^{2 M} \alpha_{m} \hat{\mathbf{U}}_{m}^{\prime}$.

For the calculation of modal coefficients, both left and right eigenvectors are needed because the system (16) is unsymmetric. Denoting $\hat{\mathbf{U}}_{m}^{\prime}$ and $\hat{\mathbf{V}}_{n}^{\prime}$ the right and left eigenvectors respectively, the following biorthogonality relations hold $[45,32,14]$ :

$$
\hat{\mathbf{V}}_{n}^{\prime T} \mathbf{B} \hat{\mathbf{U}}_{m}^{\prime}=b_{m} \delta_{m n}, \quad \hat{\mathbf{V}}_{n}^{\prime T} \mathbf{A} \hat{\mathbf{U}}_{m}^{\prime}=k_{m} b_{m} \delta_{m n}
$$

where $b_{m}$ is a normalization factor and $\delta_{m n}$ denotes Kronecker's symbol.

Using the eigenmode expansion into Eq. (16) and taking advantage of biorthogonality relations (19), the $\alpha_{m}$ 's can be determined (see for instance Refs. [36, 32, 42]). The displacement response in the wavenumber-frequency domain is then:

$$
\tilde{\mathbf{U}}^{\prime}=\sum_{m=1}^{2 M} \frac{\hat{\mathbf{V}}_{m}^{\prime T} \hat{\mathbf{F}}^{\prime}}{b_{m}\left(k_{m}-k\right)} \mathbf{U}_{m}^{\prime}
$$

The above expression can be rewritten in an interesting manner in terms of modal displacement and forces as shown in the following.

The right eigenvectors are of the form $\hat{\mathbf{U}}_{m}^{T}=\left[\begin{array}{lll}\mathbf{U}_{m}^{\prime} & k_{m} & \mathbf{U}_{m}^{\prime}\end{array}\right]^{T}$. The left eigenvectors require solving the left eigenproblem. However, it can be proved that left eigenvectors are indeed given by [14]:

$$
\hat{\mathbf{V}}_{m}^{\prime}=\left\{\begin{array}{c}
\mathbf{U}_{-m}^{\prime} \\
k_{m} \mathbf{U}_{-m}^{\prime}
\end{array}\right\}
$$

which avoids solving the left eigenproblem. Note that a given opposite-going mode $\mathbf{U}_{-m}^{\prime}$ can be directly obtained from its forward-going counterpart $\mathbf{U}_{m}^{\prime}$ thanks to the identity shown in Appendix A (from a computational point of view, this allows to further reduce the number of modes to compute).

Furthermore, the normalization coefficient can be expressed as:

$$
b_{m}=-4 k_{m} Q_{m,-m} / \omega
$$

where:

$$
Q_{m,-m}=\frac{i \omega}{4}\left(\mathbf{U}_{m}^{\prime T} \mathbf{F}_{-m}^{\prime}-\mathbf{U}_{-m}^{\prime T} \mathbf{F}_{m}^{\prime}\right)
$$

$\mathbf{F}_{m}^{\prime}$ represents the modal force associated with the displacement $\mathbf{U}_{m}^{\prime}$ and is calculated from: $\mathbf{F}_{m}^{\prime}=\left(\mathbf{K}_{2}^{T}+i k_{m} \mathbf{K}_{3}\right) \mathbf{U}_{m}^{\prime}[46$, 14].

Using Eqs. (21)-(23), the forced response (20) becomes:

$$
\tilde{\mathbf{U}}^{\prime}=-i \sum_{m=1}^{2 M} \frac{\mathbf{E}_{m}^{\prime} \tilde{\mathbf{F}}^{\prime}}{\left(k-k_{m}\right)}
$$

where:

$$
\mathbf{E}_{m}^{\prime}=\frac{i \omega}{4 Q_{m,-m}} \mathbf{U}_{m}^{\prime} \mathbf{U}_{-m}^{\prime T}
$$

As shown in Ref. [14], the above solution is general and still hold for non-propagating modes and lossy waveguides. In particular, $\mathbf{C}$ can be complex. $\mathbf{E}_{m}^{\prime}$ is the so-called excitability matrix for line sources (here, the line source is oriented along $y^{\prime}$ ).

One points out that coupling terms in $x^{\prime} y, y^{\prime} x, y^{\prime} z$ and $z y^{\prime}$ inside the matrix $\mathbf{E}_{m}^{\prime}$ vanish because modal displacement components in $\left(x^{\prime}, z\right)$ and in $y^{\prime}$ are uncoupled (see Sec. 2.3).

The expression (24) is preferred to the expression (20) because it is written only in terms of modal displacement (no left eigenvectors needed) and allows a direct analogy with a fully analytical approach as shown later. 


\subsection{Inverse transforms}

Next, the solution written in the wavenumber domain $(k, \phi)$ must be transformed back to the space domain $(x, y)$. From the convention (2), the inverse two-dimensional Fourier transform is:

$$
f(x, y)=\frac{1}{(2 \pi)^{2}} \int_{-\infty}^{+\infty} \int_{-\infty}^{+\infty} \tilde{f}\left(k_{x}, k_{y}\right) e^{i\left(k_{x} x+k_{y} y\right)} d k_{x} d k_{y}
$$

Let us introduce polar coordinates $(x, y)=(r \cos \theta, r \sin \theta)$, as shown in Fig. 1. With this change of variables as well as Eq. (4), the inverse transform can be rewritten as follows:

$$
f(r, \theta)=\frac{1}{(2 \pi)^{2}} \int_{0}^{2 \pi} \int_{0}^{+\infty} \tilde{f}(k, \phi) e^{i k r \cos (\phi-\theta)} k d k d \phi
$$

The domain of integration used above is slightly different from Ref. [40] and leads to Hankel-type solutions as shown in the following.

The components of the displacement and the excitation vectors involved in Eq. (24) are now expressed in the basis $\left(\mathbf{e}_{x^{\prime}}, \mathbf{e}_{y^{\prime}}, \mathbf{e}_{z}\right)$, which depends on $\phi$. A proper application of the inverse transform (27) requires to express vectors in a basis independent of $\phi$, namely the fixed Cartesian basis $\left(\mathbf{e}_{x}, \mathbf{e}_{y}, \mathbf{e}_{z}\right)$ or the cylindrical basis $\left(\mathbf{e}_{r}, \mathbf{e}_{\theta}, \mathbf{e}_{z}\right)$. With polar variables $(r, \theta)$, the cylindrical basis appears to be a more natural choice.

In the remainder, $\tilde{\mathbf{U}}$ and $\tilde{\mathbf{F}}$ will denote displacement and force vectors with components in the cylindrical basis. FE vectors will be partitioned as follows:

$$
\tilde{\mathbf{U}}^{\prime}=\left\{\begin{array}{c}
\tilde{\mathbf{U}}_{x^{\prime}} \\
\tilde{\mathbf{U}}_{y^{\prime}} \\
\tilde{\mathbf{U}}_{z}
\end{array}\right\}, \tilde{\mathbf{F}}^{\prime}=\left\{\begin{array}{c}
\tilde{\mathbf{F}}_{x^{\prime}} \\
\tilde{\mathbf{F}}_{y^{\prime}} \\
\tilde{\mathbf{F}}_{z}
\end{array}\right\}, \tilde{\mathbf{U}}=\left\{\begin{array}{c}
\tilde{\mathbf{U}}_{r} \\
\tilde{\mathbf{U}}_{\theta} \\
\tilde{\mathbf{U}}_{z}
\end{array}\right\}, \tilde{\mathbf{F}}=\left\{\begin{array}{c}
\tilde{\mathbf{F}}_{r} \\
\tilde{\mathbf{F}}_{\theta} \\
\tilde{\mathbf{F}}_{z}
\end{array}\right\}
$$

The following rotation matrix is introduced:

$$
\mathbf{R}_{\varphi}=\left[\begin{array}{ccc}
\cos \varphi \mathbf{I} & \sin \varphi \mathbf{I} & \mathbf{0} \\
-\sin \varphi \mathbf{I} & \cos \varphi \mathbf{I} & \mathbf{0} \\
\mathbf{0} & \mathbf{0} & \mathbf{I}
\end{array}\right]
$$

where $\varphi=\phi-\theta$. Then, expressing the solution (24) in the cylindrical basis yields:

$$
\tilde{\mathbf{U}}=-i \sum_{m=1}^{2 M} \frac{\mathbf{E}_{m} \tilde{\mathbf{F}}}{\left(k-k_{m}\right)}
$$

with $\mathbf{E}_{m}=\mathbf{R}_{\varphi}^{T} \mathbf{E}_{m}^{\prime} \mathbf{R}_{\varphi} \cdot \mathbf{E}_{m}$ is explicitly given in terms of $\varphi$ by:

$$
\mathbf{E}_{m}=\left[\begin{array}{ccc}
\mathbf{E}_{m_{x^{\prime} x^{\prime}}} \cos ^{2} \varphi+\mathbf{E}_{m_{y^{\prime} y^{\prime}}} \sin ^{2} \varphi & \left(\mathbf{E}_{m_{x^{\prime} x^{\prime}}}-\mathbf{E}_{m_{y^{\prime} y^{\prime}}}\right) \cos \varphi \sin \varphi & \mathbf{E}_{m_{x^{\prime} z}} \cos \varphi \\
\left(\mathbf{E}_{m_{x^{\prime} x^{\prime}}}-\mathbf{E}_{m_{y^{\prime} y^{\prime}}} \cos \varphi \sin \varphi\right. & \mathbf{E}_{m_{x^{\prime} x^{\prime}}} \sin ^{2} \varphi+\mathbf{E}_{m_{y^{\prime} y^{\prime}}} \cos ^{2} \varphi & \mathbf{E}_{m_{x^{\prime} z}} \sin \varphi \\
\mathbf{E}_{m_{z x^{\prime}}} \cos \varphi & \mathbf{E}_{m_{2 x^{\prime}}} \sin \varphi & \mathbf{E}_{m_{z z}}
\end{array}\right]
$$

Note that the eigenvectors $\mathbf{U}_{m}^{\prime}$ do not depend on $\phi$, and so does $\mathbf{E}_{m}^{\prime}$, thanks to the assumption of transverse isotropy about the thickness direction.

The excitation vector $\tilde{\mathbf{F}}$ generally depends on $\phi$ and must be expanded as a Fourier series:

$$
\tilde{\mathbf{F}}(k, \phi)=2 \pi \sum_{p=-\infty}^{+\infty} i^{-p} \tilde{\mathbf{F}}_{p}(k) e^{i p \phi},
$$

where the $p$ th coefficient can be calculated from:

$$
\tilde{\mathbf{F}}_{p}(k)=\frac{i^{p}}{(2 \pi)^{2}} \int_{0}^{2 \pi} \tilde{\mathbf{F}}(k, \phi) e^{-i p \phi} d \phi
$$


Given the expansion form (32), $\tilde{\mathbf{F}}_{p}(k)$ is also equal to the Hankel transform of order $p$ of $\mathbf{F}_{p}(r)$, which is defined by the expansion: $\mathbf{F}(r, \theta)=\sum_{p=-\infty}^{+\infty} \mathbf{F}_{p}(r) e^{i p \theta}$ where $\mathbf{F}_{p}(r)=\frac{1}{2 \pi} \int_{0}^{2 \pi} \mathbf{F}(r, \theta) e^{-i p \theta} d \theta$ (see Appendix B).

The expression (30) becomes:

$$
\tilde{\mathbf{U}}=-2 \pi i \sum_{m=1}^{2 M} \sum_{p=-\infty}^{+\infty} i^{-p} \frac{\mathbf{E}_{m} \tilde{\mathbf{F}}_{p}}{k-k_{m}} e^{i p \phi}
$$

Applying the inverse transform (27) to Eq. (34) yields:

$$
\mathbf{U}=-\frac{i}{2 \pi} \sum_{m=1}^{2 M} \sum_{p=-\infty}^{+\infty} i^{-p} \int_{0}^{+\infty} \int_{0}^{2 \pi} \mathbf{E}_{m} e^{i k r \cos (\phi-\theta)+i p \phi} d \phi \frac{\tilde{\mathbf{F}}_{p}}{k-k_{m}} k d k
$$

Then, the following Bessel formula is recalled:

$$
\int_{0}^{2 \pi} e^{i k r \cos \psi+i q \psi} d \psi=2 \pi i^{q} J_{q}(k r)
$$

From this formula, it can be noticed that the integrals on $k$ in Eq. (35) indeed correspond to inverse Hankel transforms, the inverse Hankel transform of order $q$ of an arbitrary function $\tilde{f}_{q}(k)$ being defined as:

$$
f(r)=\int_{0}^{+\infty} \tilde{f}_{q}(k) J_{q}(k r) k d k
$$

Now let the function $\tilde{f}_{q}(k)$ have one pole so that: $\tilde{f}_{q}(k)=\tilde{g}_{q}(k) /\left(k-k_{m}\right)$, where $\tilde{g}_{q}(k)$ is supposed to be holomorphic (no pole). The application of Cauchy residue theorem yields (see Appendix $C$ ):

$$
f(r)=i \pi k_{m} \tilde{g}_{q}\left(k_{m}\right) H_{q}^{(1)}\left(k_{m} r\right)
$$

where $H_{q}^{(1)}$ denotes the $q$ th order Hankel Function of the first kind.

Finally, the application of Eqs. (36)-(38) to the expression (35) yields after tedious calculations:

$$
\left\{\begin{array}{l}
\mathbf{U}_{r} \\
\mathbf{U}_{\theta} \\
\mathbf{U}_{z}
\end{array}\right\}=\sum_{m=1}^{M} \sum_{p=-\infty}^{+\infty}\left[\begin{array}{lll}
\mathbf{G}_{r r}^{(m, p)} & \mathbf{G}_{r \theta}^{(m, p)} & \mathbf{G}_{r z}^{(m, p)} \\
\mathbf{G}_{\theta r}^{(m, p)} & \mathbf{G}_{\theta \theta}^{(m, p)} & \mathbf{G}_{\theta z, p)}^{(m,} \\
\mathbf{G}_{z r}^{(m, p)} & \mathbf{G}_{z \theta}^{(m, p)} & \mathbf{G}_{z z}^{(m, p)}
\end{array}\right]\left\{\begin{array}{c}
\tilde{\mathbf{F}}_{p_{r}}\left(k_{m}\right) \\
\tilde{\mathbf{F}}_{p_{\theta}}\left(k_{m}\right) \\
\tilde{\mathbf{F}}_{p_{z}}\left(k_{m}\right)
\end{array}\right\} \pi k_{m} e^{i p \theta}
$$

with:

$$
\begin{array}{r}
\mathbf{G}_{r r}^{(m, p)}=\mathbf{E}_{m_{x^{\prime} x^{\prime}}} H_{p}^{(1)}\left(k_{m} r\right)-\left(\mathbf{E}_{m_{x^{\prime} x^{\prime}}}-\mathbf{E}_{m_{y^{\prime} y^{\prime}}}\right)\left(\frac{H_{p+1}^{(1)}\left(k_{m} r\right)}{k_{m} r}+p(p-1) \frac{H_{p}^{(1)}\left(k_{m} r\right)}{\left(k_{m} r\right)^{2}}\right), \\
\mathbf{G}_{r \theta}^{(m, p)}=\mathbf{G}_{\theta r}^{(m, p)}=i\left(\mathbf{E}_{m_{x^{\prime} x^{\prime}}}-\mathbf{E}_{m_{y^{\prime} y^{\prime}}}\right)\left(p \frac{H_{p+1}^{(1)}\left(k_{m} r\right)}{k_{m} r}-p(p-1) \frac{H_{p}^{(1)}\left(k_{m} r\right)}{\left(k_{m} r\right)^{2}}\right), \\
\mathbf{G}_{r z}^{(m, p)}=i \mathbf{E}_{m_{x^{\prime} z}}\left(H_{p+1}^{(1)}\left(k_{m} r\right)-p \frac{H_{p}^{(1)}\left(k_{m} r\right)}{k_{m} r}\right), \mathbf{G}_{z r}^{(m, p)}=i \mathbf{E}_{m_{z x^{\prime}}}\left(H_{p+1}^{(1)}\left(k_{m} r\right)-p \frac{H_{p}^{(1)}\left(k_{m} r\right)}{k_{m} r}\right), \\
\mathbf{G}_{\theta \theta}^{(m, p)}=\mathbf{E}_{m_{y^{\prime} y^{\prime}}} H_{p}^{(1)}\left(k_{m} r\right)-\left(\mathbf{E}_{m_{y^{\prime} y^{\prime}}}-\mathbf{E}_{m_{x^{\prime} x^{\prime}}}\right)\left(\frac{H_{p+1}^{(1)}\left(k_{m} r\right)}{k_{m} r}+p(p-1) \frac{H_{p}^{(1)}\left(k_{m} r\right)}{\left(k_{m} r\right)^{2}}\right), \\
\mathbf{G}_{\theta z}^{(m, p)}=\mathbf{E}_{m_{x^{\prime} z} z} p \frac{H_{p}^{(1)}\left(k_{m} r\right)}{k_{m} r}, \mathbf{G}_{z \theta}^{(m, p)}=\mathbf{E}_{m_{z x^{\prime}}} p \frac{H_{p}^{(1)}\left(k_{m} r\right)}{k_{m} r}, \mathbf{G}_{z z}^{(m, p)}=\mathbf{E}_{m_{z z}} H_{p}^{(1)}\left(k_{m} r\right)
\end{array}
$$

For clarity, the calculation of $\mathbf{G}_{r r}^{(m, p)}$ is detailed in Appendix D. The other components of $\mathbf{G}^{(m, p)}$ can be obtained in the same manner. The identities $\mathbf{G}_{r \theta}^{(m, p)}=\mathbf{G}_{\theta r}^{(m, p)^{T}}, \mathbf{G}_{r z}^{(m, p)}=-\mathbf{G}_{z r}^{(m, p)^{T}}$ and $\mathbf{G}_{\theta z}^{(m, p)}=-\mathbf{G}_{z \theta}^{(m, p)^{T}}$ result from the reciprocity principle. The terms of order $1 / k_{m} r$ and $\left(1 / k_{m} r\right)^{2}$ represent near field contributions and can be negligible in the far-field.

In practice, the sum over the $M$ guided modes is truncated [14] (one retains less modes than the number of dofs), as well as the infinite sum over the Fourier coefficients $p$. The response in the time domain can finally be obtained from the inverse time Fourier transform of $\mathbf{U}$, given by $\frac{1}{2 \pi} \int_{-\infty}^{+\infty} \mathbf{U} e^{-i \omega} d \omega$. 
In order for the waves to be diverging, it is emphasized that only the positive-going modes must be retained in the expansion (this results from the proper application of the Cauchy residue theorem). As a consequence, the sum in Eq. (39) is over the positive-going modes, excluding the negative-going ones. Note that one must be careful to select the proper backward modes (if any), having positive energy velocities but negative phase velocities. Similarly to the 2D modeling of Lamb waves [14], the expansion (39) is valid for any point lying outside the source region, i.e. such that $r>r_{s}$ where $r_{s}$ denotes the maximum distance at which the excitation $\mathbf{F}(r, \theta)$ is non-zero.

One recalls that the sum over $m$ in Eq. (39) can be decomposed into a sum over Lamb modes, satisfying $\mathbf{E}_{m_{y^{\prime} y^{\prime}}}=\mathbf{0}$, and a sum over SH modes, for which the only non zero component is $\mathbf{E}_{m_{y^{\prime} y^{\prime}}}$ (as already mentioned, this decomposition is not applied here in order to keep compact expressions). Then from Eq. (39), it can be checked that a force normal to the plate (i.e. along $z$ ) does not generate any SH modes for any $p$. Note that the expression of $\mathbf{G}_{z z}^{(m, p)}$ is quite simple and yields a straightforward solution for the normal displacement excited by a force normal to the plate.

\subsection{Expression with force components in the Cartesian basis}

For sources defined in the Cartesian coordinate system, it is useful to rewrite the previous solution in terms of force components expressed in the Cartesian basis instead of the cylindrical one. One has:

$$
\left\{\begin{array}{c}
\tilde{\mathbf{F}}_{p_{r}} \\
\tilde{\mathbf{F}}_{p_{\theta}} \\
\tilde{\mathbf{F}}_{p_{z}}
\end{array}\right\}=\left[\begin{array}{ccc}
\cos \theta \mathbf{I} & \sin \theta \mathbf{I} & \mathbf{0} \\
-\sin \theta \mathbf{I} & \cos \theta \mathbf{I} & \mathbf{0} \\
\mathbf{0} & \mathbf{0} & \mathbf{I}
\end{array}\right]\left\{\begin{array}{c}
\tilde{\mathbf{F}}_{p_{x}} \\
\tilde{\mathbf{F}}_{p_{y}} \\
\tilde{\mathbf{F}}_{p_{z}}
\end{array}\right\}
$$

Using Eq. (41) into Eq. (39) yields after calculations:

$$
\left\{\begin{array}{l}
\mathbf{U}_{r} \\
\mathbf{U}_{\theta} \\
\mathbf{U}_{z}
\end{array}\right\}=\sum_{m=1}^{M} \sum_{p=-\infty}^{+\infty}\left[\begin{array}{lll}
\mathbf{G}_{r x}^{(m, p)} & \mathbf{G}_{r y}^{(m, p)} & \mathbf{G}_{r z}^{(m, p)} \\
\mathbf{G}_{\theta x}^{(m, p)} & \mathbf{G}_{\theta y}^{(m, p)} & \mathbf{G}_{\theta z}^{(m, p)} \\
\mathbf{G}_{z x}^{(m, p)} & \mathbf{G}_{z y}^{(m, p)} & \mathbf{G}_{z z}^{(m, p)}
\end{array}\right]\left\{\begin{array}{c}
\tilde{\mathbf{F}}_{p_{x}}\left(k_{m}\right) \\
\tilde{\mathbf{F}}_{p_{y}}\left(k_{m}\right) \\
\tilde{\mathbf{F}}_{p_{z}}\left(k_{m}\right)
\end{array}\right\} \pi k_{m} e^{i p \theta}
$$

with:

$$
\begin{aligned}
& \mathbf{G}_{r x}^{(m, p)}=\mathbf{E}_{m_{x^{\prime} x^{\prime}}} H_{p}^{(1)}\left(k_{m} r\right) \cos \theta-\frac{\mathbf{E}_{m_{x^{\prime} x^{\prime}}}-\mathbf{E}_{m_{y^{\prime} y^{\prime}}}}{2}\left((p+1) \frac{H_{p+1}^{(1)}\left(k_{m} r\right)}{k_{m} r} e^{i \theta}+(p-1) \frac{H_{p-1}^{(1)}\left(k_{m} r\right)}{k_{m} r} e^{-i \theta}\right), \\
& \mathbf{G}_{r y}^{(m, p)}=\mathbf{E}_{m_{x^{\prime} x^{\prime}}} H_{p}^{(1)}\left(k_{m} r\right) \sin \theta+i \frac{\mathbf{E}_{m_{x^{\prime} x^{\prime}}}-\mathbf{E}_{m_{y^{\prime} y^{\prime}}}}{2}\left((p+1) \frac{H_{p+1}^{(1)}\left(k_{m} r\right)}{k_{m} r} e^{i \theta}-(p-1) \frac{H_{p-1}^{(1)}\left(k_{m} r\right)}{k_{m} r} e^{-i \theta),}\right. \\
& \mathbf{G}_{\theta x}^{(m, p)}=-\mathbf{E}_{m_{y^{\prime} y^{\prime}}} H_{p}^{(1)}\left(k_{m} r\right) \sin \theta+i \frac{\mathbf{E}_{m_{x^{\prime} x^{\prime}}}-\mathbf{E}_{m_{y^{\prime} y^{\prime}}}}{2}\left((p+1) \frac{H_{p+1}^{(1)}\left(k_{m} r\right)}{k_{m} r} e^{i \theta}-(p-1) \frac{H_{p-1}^{(1)}\left(k_{m} r\right)}{k_{m} r} e^{-i \theta}\right), \\
& \mathbf{G}_{\theta y}^{(m, p)}=\mathbf{E}_{m_{y^{\prime} y^{\prime}}} H_{p}^{(1)}\left(k_{m} r\right) \cos \theta+\frac{\mathbf{E}_{m_{x^{\prime} x^{\prime}}}-\mathbf{E}_{m_{y^{\prime} y^{\prime}}}}{2}\left((p+1) \frac{H_{p+1}^{(1)}\left(k_{m} r\right)}{k_{m} r} e^{i \theta}+(p-1) \frac{H_{p-1}^{(1)}\left(k_{m} r\right)}{k_{m} r} e^{-i \theta),}\right. \\
& \mathbf{G}_{z x}^{(m, p)}=i \mathbf{E}_{m_{z x^{\prime}}}\left(H_{p+1}^{(1)}\left(k_{m} r\right) \cos \theta-p \frac{H_{p}^{(1)}\left(k_{m} r\right)}{k_{m} r} e^{-i \theta}\right), \\
& \mathbf{G}_{z y}^{(m, p)}=i \mathbf{E}_{m_{z x^{\prime}}}\left(H_{p+1}^{(1)}\left(k_{m} r\right) \sin \theta-i p \frac{H_{p}^{(1)}\left(k_{m} r\right)}{k_{m} r} e^{-i \theta}\right)
\end{aligned}
$$

Equations (39), (40), (42) and (43) are the central results of this paper. One points out that no specific assumption has been made except transverse isotropy and invariance of material properties in the $(x, y)$ plane. The solutions remain applicable when $\mathbf{C}$ is complex (case of viscoelastic materials) and when the properties arbitrarily depend on $z$. Also, the modal expansions account for the near field contribution, resulting from terms in $1 / k_{m} r$ as well as non-propagating modes, evanescent or inhomogeneous. The modal responses thus remain applicable close to the source (provided that the truncated expansions include enough modes).

\subsection{Expressions in the far field}

In the far field, the following asymptotic expression holds:

$$
H_{p}^{(1)}\left(k_{m} r\right)=\sqrt{\frac{2}{\pi k_{m} r}} e^{i\left(k_{m} r-\left(p+\frac{1}{2}\right) \frac{\pi}{2}\right)}+O\left(r^{-3 / 2}\right)
$$


In the solution (39), the sums over $p$ are in the form:

$$
\sum_{p=-\infty}^{+\infty} \pi k_{m} \tilde{\mathbf{F}}_{p}\left(k_{m}\right) H_{p}^{(1)}\left(k_{m} r\right) e^{i p \theta}
$$

Thanks to the identity $\sum i^{-p} \tilde{\mathbf{F}}_{p}\left(k_{m}\right) e^{i p \theta}=\tilde{\mathbf{F}}\left(k_{m}, \theta\right) / 2 \pi$ (see Eq. (32)), the use of Eq. (44) into (45) yields:

$$
\sqrt{\frac{k_{m}}{2 \pi}} e^{-i \pi / 4} \frac{e^{i k_{m} r}}{\sqrt{r}} \tilde{\mathbf{F}}\left(k_{m}, \theta\right)+O\left(r^{-3 / 2}\right)
$$

This avoids to perform the sum over $p$.

The expression (39) can then be remarkably simplified in the far field as:

$$
\left\{\begin{array}{l}
\mathbf{U}_{r} \\
\mathbf{U}_{\theta} \\
\mathbf{U}_{z}
\end{array}\right\}=\sum_{m=1}^{M} \sqrt{\frac{k_{m}}{2 \pi}} e^{-i \pi / 4} \mathbf{E}_{m}^{\prime} \tilde{\mathbf{F}}\left(k_{m}, \theta\right) \frac{e^{i k_{m} r}}{\sqrt{r}}+O\left(r^{-3 / 2}\right)
$$

It should be kept in mind that $\tilde{\mathbf{F}}=\left[\begin{array}{lll}\tilde{\mathbf{F}}_{r} & \tilde{\mathbf{F}}_{\theta} & \tilde{\mathbf{F}}_{z}\end{array}\right]^{T}$ is expressed in the cylindrical basis. For a force expressed in the Cartesian basis, the above far field approximation holds by replacing $\tilde{\mathbf{F}}$ with $\mathbf{R}_{\theta}\left[\tilde{\mathbf{F}}_{x} \tilde{\mathbf{F}}_{y} \tilde{\mathbf{F}}_{z}\right]^{T}$.

\subsection{Fully analytical solution}

The solutions presented insofar are semi-analytical in the sense that the $z$ direction has been discretized with finite elements. These expressions can be readily rewritten in a fully analytical form. From Eqs. (10) and (14), it can be shown that the following identity holds:

$$
\delta \tilde{\mathbf{U}}^{\prime T} \tilde{\mathbf{F}}^{\prime}=\int_{z} \delta \mathbf{u}^{\prime T} \mathbf{f}^{\prime} d z+\left[\delta \mathbf{u}^{\prime T} \mathbf{t}^{\prime}\right]_{z_{1}}^{z_{2}}
$$

where $z=z_{1}$ and $z=z_{2}$ are the plate boundaries. For brevity, let us consider the solution (42) (the solution (39) can be handled in the same manner). The discretized terms are written under the form:

$$
\mathbf{E}_{m_{i^{\prime} j^{\prime}}} \tilde{\mathbf{F}}_{p_{l}}=\frac{i \omega}{4 Q_{m,-m}} \mathbf{U}_{m_{i^{\prime}}} \alpha_{j^{\prime} l}^{(-m, p)}, \quad \text { with: } \alpha_{j^{\prime} l}^{(-m, p)}=\mathbf{U}_{-m_{j^{\prime}}}^{T} \tilde{\mathbf{F}}_{p_{l}}
$$

where $i^{\prime}=\left(x^{\prime}, y^{\prime}, z\right), j^{\prime}=\left(x^{\prime}, y^{\prime}, z\right)$ and $l=(x, y, z)$.

The fully analytical solution, written in terms of $(r, \theta, z ; \omega)$, can be deduced from its discretized counterpart (42) thanks to the following substitutions:

$$
\begin{array}{r}
{\left[\mathbf{U}_{r} \mathbf{U}_{\theta} \mathbf{U}_{z}\right]^{T} \longrightarrow\left[\begin{array}{lll}
u_{r} & u_{\theta} & u_{z}
\end{array}\right]^{T},} \\
\mathbf{E}_{m_{i^{\prime} j^{\prime}}} \tilde{\mathbf{F}}_{p_{l}}\left(k_{m}\right) \longrightarrow \frac{i \omega}{4 Q_{m,-m}} u_{m_{i^{\prime}}}(z) \alpha_{j^{\prime} l}^{(-m, p)}\left(k_{m}\right)
\end{array}
$$

where from Eq. (48), one has:

$$
\alpha_{j^{\prime} l}^{(-m, p)}\left(k_{m}\right)=\int_{z} u_{-m_{j^{\prime}}}(z) \tilde{f}_{p_{l}}\left(z ; k_{m}\right) d z+\left[u_{-m_{j^{\prime}}}(z) \tilde{t}_{p_{l}}\left(z ; k_{m}\right)\right]_{z_{1}}^{z_{2}}
$$

and from Ref. [14]:

$$
Q_{m,-m}=\frac{i \omega}{4} \int_{z}\left(\mathbf{u}_{m}^{\prime} \cdot \mathbf{t}_{-m}^{\prime}-\mathbf{u}_{-m}^{\prime} \cdot \mathbf{t}_{m}^{\prime}\right) d z
$$

with $t_{i^{\prime}}=\sigma_{i^{\prime} x^{\prime}}$.

Note that a fully analytical approach has the advantage to be exact in the frequency-wavenumber domain [38, 39, $6,7]$. However, each layer must be homogeneous and complex root finding algorithms are usually required to get modal wavenumbers. 


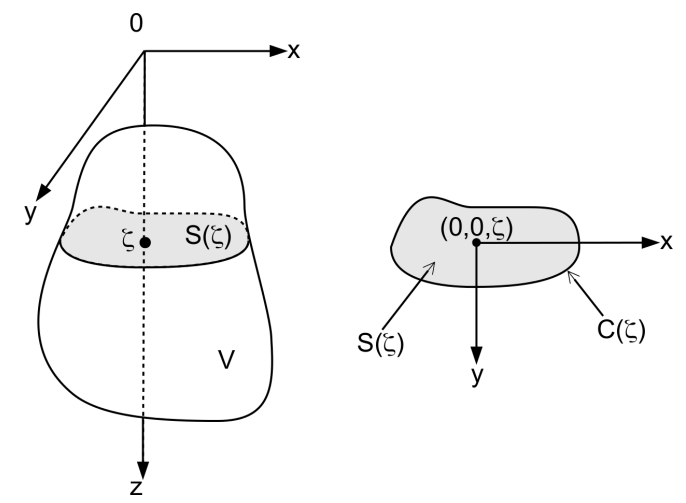

Figure 2: Example of an internal three-dimensional source of complex shape. $V$ denotes the source region. The plane surface $S(\zeta)$ corresponds to the intersection of the three-dimensional region $V$ and the horizontal plane $z=\zeta$. The curve $C(\zeta)$ delimits the surface $S(\zeta)$.

\subsection{Source term calculation}

The method is applicable to internal or surface sources of arbitrary shape. For clarity, Fig. 2 depicts the example of an internal source $\mathbf{f}(x, y, z ; \omega)$ localized into a finite three-dimensional region $V$. We drop the $\omega$ dependence for conciseness. One assumes that $\mathbf{f}(x, y, z)$ is expressed in the Cartesian basis. Let us consider that the source region $V$ is delimited by a surface parametrized by the implicit equation $s(x, y, z)=0$ so that $V=\left\{(x, y, z) \in \mathbb{R}^{3}: s(x, y, z) \leq 0\right\}$.

In the modal solution of this paper, the volume source term appears as the vector function $\tilde{\mathbf{f}}_{p}(z ; k)$ evaluated at $k=k_{m}$, which can be calculated by two methods. From Eqs. (2) and (33), $\tilde{\mathbf{f}}_{p}(z ; k)$ is equal to the $p$ th Fourier coefficient of the spatial two-dimensional Fourier Transform of $\mathbf{f}(x, y, z)$ and can be obtained from the following triple integral:

$$
\tilde{\mathbf{f}}_{p}(z ; k)=\frac{i^{p}}{(2 \pi)^{2}} \int_{0}^{2 \pi} \iint_{S(z)} \mathbf{f}(x, y, z) e^{-i(k x \cos \phi+k y \sin \phi+p \phi)} d x d y d \phi
$$

where $S(\zeta)=\left\{(x, y) \in \mathbb{R}^{2}: s(x, y, \zeta) \leq 0\right\}$ is the plane surface resulting from the intersection of the volume $V$ and the horizontal plane $z=\zeta$. The equation $s(x, y, \zeta)=0$ parametrizes the curve $C(\zeta)$ enclosing $S(\zeta)$ (see Fig. 2). As proved in Appendix B, $\tilde{\mathbf{f}}_{p}(z ; k)$ is also equal to the Hankel transform of order $p$ of the $p$ th Fourier coefficient of $\mathbf{f}(x, y, z)=\mathbf{f}(r \cos \theta, r \sin \theta, z)$, yielding the double integral:

$$
\tilde{\mathbf{f}}_{p}(z ; k)=\frac{1}{2 \pi} \iint_{S(z)} \mathbf{f}(r \cos \theta, r \sin \theta, z) J_{p}(k r) e^{-i p \theta} r d r d \theta
$$

where $S(\zeta)=\left\{(r, \theta) \in \mathbb{R}^{+} \times[0,2 \pi]: s(r \cos \theta, r \sin \theta, \zeta) \leq 0\right\}$. From a mathematical point of view, both expressions give the same result. However in case of numerical integration, the expression (53) can suffer from round-off errors as $p$ increases (see Sec. 5).

The numerical evaluation of these multiple integrals for each value of $k_{m}$ might be relatively costly compared to the other fast operations required by the modal solutions of this paper. This cost can yet be reduced by carrying out analytical calculations as far as possible (see example in Sec. 5). Note that with a SAFE method, internal sources of complex shape must be carefully handled. In general, the SAFE force vector must be recomputed for each $k_{m}$ from the following elementary vector:

$$
\left\{\begin{array}{c}
\tilde{\mathbf{F}}_{p_{x}}^{e}\left(k_{m}\right) \\
\tilde{\mathbf{F}}_{p_{y}}^{e}\left(k_{m}\right) \\
\tilde{\mathbf{F}}_{p_{z}}^{e}\left(k_{m}\right)
\end{array}\right\}=\int_{z} \mathbf{N}^{e T} \tilde{\mathbf{f}}_{p}\left(z ; k_{m}\right) d z
$$

Surface sources $\mathbf{t}(x, y ; \omega)$ (applied tractions) are treated in the same way as internal sources, the only difference being that such source terms do not depend on $z$ and are hence simpler to implement. 


\section{Validation with literature results}

\subsection{Solution for a point source}

Let us consider a source concentrated at $r=0$. The source can be arbitrarily distributed along the $z$ direction. The force vector has the following form:

$$
\mathbf{f}(x, y, z ; \omega)=\left(f_{x}(z ; \omega) \mathbf{e}_{x}+f_{y}(z ; \omega) \mathbf{e}_{y}+f_{z}(z ; \omega) \mathbf{e}_{z}\right) \delta(x) \delta(y)
$$

where $f_{x}, f_{y}$ and $f_{z}$ denotes the force components in the Cartesian basis. The corresponding discretized FE force is:

$$
\left\{\begin{array}{l}
\mathbf{F}_{x}(x, y) \\
\mathbf{F}_{y}(x, y) \\
\mathbf{F}_{z}(x, y)
\end{array}\right\}=\delta(x) \delta(y)\left\{\begin{array}{l}
\mathbf{F}_{x} \\
\mathbf{F}_{y} \\
\mathbf{F}_{z}
\end{array}\right\}
$$

The $\omega$ dependence is dropped for conciseness.

The source expression in cylindrical coordinates is readily obtained by substituting $\delta(x) \delta(y)$ with $\delta(r) / 2 \pi r$ [47], which does not depend on $\theta$. Hence, $\mathbf{F}_{0}=\mathbf{F}$ and $\mathbf{F}_{p}=\mathbf{0}$ for $p \neq 0$ (the only remaining term in the sum over $m$ in Eq. (42) is $p=0)$. The Hankel transform of order 0 of $\delta(r) / 2 \pi r$ is equal to $1 / 2 \pi$, which yields:

$$
\left\{\begin{array}{c}
\tilde{\mathbf{F}}_{0_{x}}(k) \\
\tilde{\mathbf{F}}_{y_{y}}(k) \\
\tilde{\mathbf{F}}_{0_{z}}(k)
\end{array}\right\}=\frac{1}{2 \pi}\left\{\begin{array}{l}
\mathbf{F}_{x} \\
\mathbf{F}_{y} \\
\mathbf{F}_{z}
\end{array}\right\}
$$

Now, the sum over $m$ in Eq. (42) is decomposed into a sum over Lamb modes, satisfying $\mathbf{E}_{m_{\gamma^{\prime} \gamma^{\prime}}}=\mathbf{0}$, and a sum over $\mathrm{SH}$ modes, for which the only non zero component is $\mathbf{E}_{m_{\gamma^{\prime} \gamma^{\prime}}}$. After rearrangements, the solution (42) finally becomes:

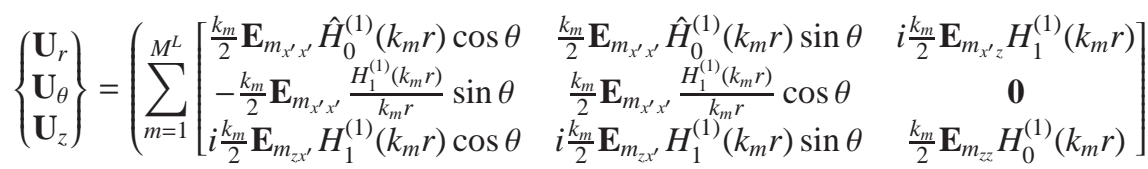

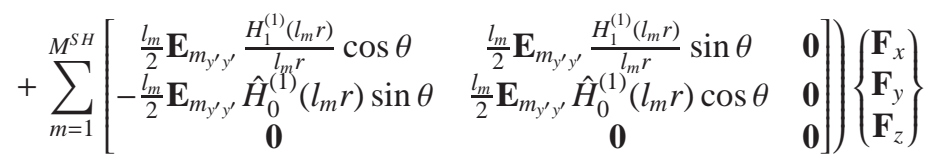

with the notation $\hat{H}_{0}^{(1)}(\cdot)=H_{0}^{(1)}(\cdot)-\frac{H_{1}^{(1)}(\cdot)}{(\cdot)} . \quad k_{m}$ and $l_{m}$ denote the wavenumbers of Lamb modes and SH modes respectively.

As a side remark, a force directed along $z$ does not generate any displacement component in $\theta$ for $p=0$. This is not true for $p \neq 0$ as shown by the expression of $\mathbf{G}_{\theta z}^{(m, p)}$.

\subsection{Interpretation in terms of excitabilities}

Each component in the two matrices of Eq. (59), associated with Lamb and SH modes respectively, involves a product of three terms:

- the first term, given by the product between $\mathbf{E}_{m_{i^{\prime} j^{\prime}}}$ and $\pm \frac{k_{m}}{2}$ or $\pm \frac{l_{m}}{2}$ (possibly $\times i$ ), can be viewed as the modal excitability for point sources, as explained below;

- the second term, given by $\hat{H}_{0}^{(1)}\left(k_{m} r\right)$ or $\frac{H_{1}^{(1)}\left(k_{m} r\right)}{k_{m} r}$, represents the propagator (it gives the radial dependence of the field) including the near field effect, in $1 / k_{m} r$;

- the third term gives the directivity $(1, \sin \theta$ or $\cos \theta)$. 
The excitability of a mode is defined as the ratio of the displacement of that mode to a point force applied in a given direction $[11,12,13]$. With a SAFE method, the excitability becomes a matrix whose components represents the modal displacement dofs when a unit force acts at a single dof [14]. As already mentioned in Sec. 3.1, $\mathbf{E}_{m_{i^{\prime} j^{\prime}}}$ is equal to the excitability matrix for line sources (along the $y^{\prime}$ direction). Hence, Eq. (59) indeed gives useful formula to calculate the excitabilities of point sources from lines sources. One emphasizes that these excitabilities and propagators remain valid for non-propagating modes, viscoelastic materials and include the near-field effect.

For instance, the radial displacement generated by a force in the $x$ direction $\mathbf{F}_{x}$ is expressed as:

$$
\mathbf{U}_{r}=\sum_{m=1}^{M^{L}} \frac{k_{m}}{2} \mathbf{E}_{m_{x^{\prime} x^{\prime}}} \mathbf{F}_{x} \hat{H}_{0}^{(1)}\left(k_{m} r\right) \cos \theta+\sum_{m=1}^{M^{S H}} \frac{l_{m}}{2} \mathbf{E}_{m_{y^{\prime} y^{\prime}}} \mathbf{F}_{x} \frac{H_{1}^{(1)}\left(l_{m} r\right)}{l_{m} r} \cos \theta
$$

In the above expression, $\frac{k_{m}}{2} \mathbf{E}_{m_{x^{\prime} x^{\prime}}}$ and $\frac{l_{m}}{2} \mathbf{E}_{m_{y^{\prime} y^{\prime}}}$ are the radial excitabilities due to point forces in the $x$ direction, for Lamb and $\mathrm{SH}$ modes respectively.

Similarly, the excitability for a displacement and a point force both directed along $z$ is given by $\frac{k_{m}}{2} \mathbf{E}_{m_{z z}}$ for Lamb modes (the propagator being $H_{0}^{(1)}\left(k_{m} r\right)$ ), and is $\mathbf{0}$ for SH modes. This result is checked by comparing the solutions of Viktorov [2] (for line sources) and Ditri [18] (for point sources).

Following the work of Velichko et al. [13], one can also define modal excitabilities in the far field, with propagators in $e^{i k_{m} r} / \sqrt{r}$. If the displacement and the force vectors are both expressed in the Cartesian basis, Eq. (47) yields:

$$
\left\{\begin{array}{l}
\mathbf{U}_{x} \\
\mathbf{U}_{y} \\
\mathbf{U}_{z}
\end{array}\right\}=\sum_{m=1}^{M} \sqrt{\frac{k_{m}}{2 \pi}} e^{-i \pi / 4} \mathbf{R}_{\theta}^{T} \mathbf{E}_{m}^{\prime} \mathbf{R}_{\theta} \frac{e^{i k_{m} r}}{\sqrt{r}}\left\{\begin{array}{l}
\mathbf{F}_{x} \\
\mathbf{F}_{y} \\
\mathbf{F}_{z}
\end{array}\right\}+O\left(r^{-3 / 2}\right)
$$

This result precisely corresponds to the discretized version of the relation found in Ref. [13] for the special case of an isotropic or transversely isotropic layered medium. The slight difference with Ref. [13] is that, in this paper, Eq. (61) constitutes a generalization to lossy waveguides and to any kind of modes, propagating or not, because the line source excitability defined by Eq. (25) is not restricted to propagating modes in lossless waveguides [14].

\subsection{Comparison with Achenbach's result}

In Refs. [9, 26], a modal solution had been proposed for the wave motion of an isotropic layer generated by a point load of arbitrary direction. The approach of Achenbach and co-workers is fully analytical, based on the concept of carrier waves [25] together with a novel application of elastodynamic reciprocity based on appropriate dummy solutions.

First, let us rewrite the solution proposed in the present paper. Using Eqs. (A.2) and (A.4) into Eq. (23), one has:

$$
Q_{m,-m}=\frac{\omega}{2} I_{m m}
$$

with the notation:

$$
I_{m m}=i\left(\mathbf{U}_{m_{z}}^{T} \mathbf{F}_{m_{z}}-\mathbf{U}_{m_{x^{\prime}}}^{T} \mathbf{F}_{m_{x^{\prime}}}-\mathbf{U}_{m_{y^{\prime}}}^{T} \mathbf{F}_{m_{y^{\prime}}}\right)
$$

Using Eqs. (A.2) and (62) into Eq. (25), the line source excitabilities can be rewritten as follows:

$$
\begin{aligned}
\mathbf{E}_{m_{x^{\prime} x^{\prime}}}=i \frac{\mathbf{U}_{m_{x^{\prime}}} \mathbf{U}_{m_{x^{\prime}}}^{T}}{2 I_{m m}}, \quad \mathbf{E}_{m_{x^{\prime} z}} & =-i \frac{\mathbf{U}_{m_{x^{\prime}}} \mathbf{U}_{m_{z}}^{T}}{2 I_{m m}}, \quad \mathbf{E}_{m_{z x^{\prime}}}=i \frac{\mathbf{U}_{m_{z}} \mathbf{U}_{m_{x^{\prime}}}^{T}}{2 I_{m m}}, \\
\mathbf{E}_{m_{z z}} & =-i \frac{\mathbf{U}_{m_{z}} \mathbf{U}_{m_{z}}^{T}}{2 I_{m m}}, \quad \mathbf{E}_{m_{y^{\prime} y^{\prime}}}=i \frac{\mathbf{U}_{m_{y^{\prime}}} \mathbf{U}_{m_{y^{\prime}}}^{T}}{2 I_{m m}}
\end{aligned}
$$

Note that $\mathbf{E}_{m_{x^{\prime} x^{\prime}}}^{T}=\mathbf{E}_{m_{x^{\prime} x^{\prime}}}, \mathbf{E}_{m_{z z}}^{T}=\mathbf{E}_{m_{z z}}, \mathbf{E}_{m_{y^{\prime} y^{\prime}}}^{T}=\mathbf{E}_{m_{y^{\prime} y^{\prime}}}$ and $\mathbf{E}_{m_{x^{\prime} z}}^{T}=-\mathbf{E}_{m_{z x^{\prime}}}$. These symmetry properties are a consequence of the reciprocity principle.

Let us consider a point source localized at $z=z_{0}$. In Eq. (56), the force components are now given by: $f_{l}(z ; \omega)=$ $f_{l}(\omega) \delta\left(z-z_{0}\right)$. From Sec. 3.5, one has $\alpha_{j^{\prime} l}^{(-m, 0)}\left(k_{m}\right)=u_{-m_{j^{\prime}}}\left(z_{0}\right) f_{l}(\omega) / 2 \pi$, and the fully analytical forms of the line source 
excitabilities given by Eq. (64) are:

$$
\begin{aligned}
e_{m_{x^{\prime} x^{\prime}}}=i \frac{u_{m_{x^{\prime}}}(z) u_{m_{x^{\prime}}}\left(z_{0}\right)}{2 I_{m m}}, e_{m_{x^{\prime} z}}=-i \frac{u_{m_{x^{\prime}}}(z) u_{m_{z}}\left(z_{0}\right)}{2 I_{m m}}, e_{m_{z^{\prime}}}=i \frac{u_{m_{z}}(z) u_{m_{x^{\prime}}}\left(z_{0}\right)}{2 I_{m m}}, \\
e_{m_{z z}}=-i \frac{u_{m_{z}}(z) u_{m_{z}}\left(z_{0}\right)}{2 I_{m m}}, e_{m_{y^{\prime} y^{\prime}}}=i \frac{u_{m_{y^{\prime}}}(z) u_{m_{y^{\prime}}}\left(z_{0}\right)}{2 I_{m m}}
\end{aligned}
$$

with:

$$
I_{m m}=i \int_{z}\left(u_{m_{z}} \sigma_{m_{2 x^{\prime}}}-u_{m_{x^{\prime}}} \sigma_{m_{x^{\prime} x^{\prime}}}-u_{m_{y^{\prime}}} \sigma_{m_{y^{\prime} x^{\prime}}}\right) d z
$$

For clarity, the displacement and stress components are redenoted in Achenbach's work as follows: $u_{x^{\prime}}=i V, u_{z}=W$, $\sigma_{x^{\prime} x^{\prime}}=T_{11}, \sigma_{z x^{\prime}}=i T_{1 z}, u_{y^{\prime}}=U$ and $\sigma_{y^{\prime} x^{\prime}}=T_{12}$. Then, it can be checked that the analytical form associated with Eq. (59) agrees with the solution of Refs. [9, 26], obtained with $f_{y}=0$ for an isotropic plate. Reciprocally, this somewhat shows that the elegant approach followed by Achenbach and his co-workers - though not fully justified by mathematical considerations as stated in Ref. [48] - indeed leads to exact results, applicable in the near field, in the viscoelastic case and to non-propagating modes.

\subsection{Case of rectangular sources}

Let us assume an excitation represented by separable variable functions:

$$
\left\{\begin{array}{l}
\mathbf{F}_{x}(x, y ; \omega) \\
\mathbf{F}_{y}(x, y ; \omega) \\
\mathbf{F}_{z}(x, y ; \omega)
\end{array}\right\}=X(x) Y(y)\left\{\begin{array}{l}
\mathbf{F}_{x}(\omega) \\
\mathbf{F}_{y}(\omega) \\
\mathbf{F}_{z}(\omega)
\end{array}\right\}
$$

Such an assumption is often applicable for rectangular sources [28]. Dropping the $\omega$ dependence for conciseness, the 2D Fourier transform of the excitation is:

$$
\left\{\begin{array}{l}
\tilde{\mathbf{F}}_{x}\left(k_{x}, k_{y}\right) \\
\tilde{\mathbf{F}}_{y}\left(k_{x}, k_{y}\right) \\
\tilde{\mathbf{F}}_{z}\left(k_{x}, k_{y}\right)
\end{array}\right\}=\tilde{X}\left(k_{x}\right) \tilde{Y}\left(k_{y}\right)\left\{\begin{array}{l}
\mathbf{F}_{x} \\
\mathbf{F}_{y} \\
\mathbf{F}_{z}
\end{array}\right\}=\tilde{X}(k \cos \phi) \tilde{Y}(k \sin \phi)\left\{\begin{array}{l}
\mathbf{F}_{x} \\
\mathbf{F}_{y} \\
\mathbf{F}_{z}
\end{array}\right\}
$$

Then in the far field, Eq. (47) can be rewritten as:

$$
\left\{\begin{array}{l}
\mathbf{U}_{r} \\
\mathbf{U}_{\theta} \\
\mathbf{U}_{z}
\end{array}\right\}=\sum_{m=1}^{M} \sqrt{\frac{k_{m}}{2 \pi}} e^{-i \pi / 4} \tilde{Y}\left(k_{m} \sin \theta\right) \mathbf{U}_{m}^{2 D} \frac{e^{i k_{m} r}}{\sqrt{r}}+O\left(r^{-3 / 2}\right)
$$

with:

$$
\mathbf{U}_{m}^{2 D}=\tilde{X}\left(k_{m} \cos \theta\right) \mathbf{E}_{m}^{\prime} \mathbf{R}_{\theta}\left\{\begin{array}{l}
\mathbf{F}_{x} \\
\mathbf{F}_{y} \\
\mathbf{F}_{z}
\end{array}\right\}
$$

$\mathbf{U}_{m}^{2 D}$ can be viewed as the modal contribution calculated from a 2D line source problem (i.e. with infinite length excitation along $y^{\prime}$ ). For the $z$ displacement excited by a force along $z$, Eq. (69) coincides with the result of Moulin et al. [28]. The solution (69) can be viewed as a generalization to any displacement component excited by sources of arbitrary direction.

As an example, let us consider a unit excitation uniform over the directions $x$ and $y$, over finite lengths $a$ and $b$ respectively. Then, the Fourier transforms are given by: $\tilde{X}(k \cos \phi)=2 \sin \left(\frac{k a}{2} \cos \phi\right) / k \cos \phi$ and $\tilde{Y}(k \sin \phi)=$ $2 \sin \left(\frac{k b}{2} \sin \phi\right) / k \sin \phi$. If attention is restricted to the far field, then Eq. (69) can be used. If one is interested in the near field, then there is a priori no other way than using the general solution (42). In this case, the source term can be calculated from the expression (33), yielding:

$$
\left\{\begin{array}{c}
\tilde{\mathbf{F}}_{p_{x}}(k) \\
\tilde{\mathbf{F}}_{p_{y}}(k) \\
\tilde{\mathbf{F}}_{p_{z}}(k)
\end{array}\right\}=\tilde{f}_{p}(k)\left\{\begin{array}{l}
\mathbf{F}_{x} \\
\mathbf{F}_{y} \\
\mathbf{F}_{z}
\end{array}\right\}
$$


where:

$$
\tilde{f}_{p}(k)=\frac{i^{p}}{(2 \pi)^{2}} \int_{0}^{2 \pi} \frac{4 \sin \left(\frac{k a}{2} \cos \phi\right) \sin \left(\frac{k b}{2} \sin \phi\right)}{k^{2} \cos \phi \sin \phi} e^{-i p \phi} d \phi
$$

Note that the above expression, which derives from Eq. (53), is not recommended for numerical integration as discussed in Sec. 5.

\subsection{Note on the orthogonality relation used in this paper}

This subsection aims at clarifying the reason why the SAFE approach corresponds to a discretized version of a fully analytical approach. The fundamental property used in the SAFE approach is the discrete biorthogonality relation (19). As shown in Ref. [14], the relation $\hat{\mathbf{V}}_{n}^{\prime T} \mathbf{B} \hat{\mathbf{U}}_{m}^{\prime}=b_{m} \delta_{m n}$ can be rewritten as:

$$
\frac{i \omega}{4}\left(\mathbf{U}_{m}^{\prime T} \mathbf{F}_{-n}^{\prime}-\mathbf{U}_{-n}^{\prime T} \mathbf{F}_{m}^{\prime}\right)=Q_{m,-m} \delta_{m n}
$$

and the corresponding analytical form is:

$$
Q_{m,-n}=\frac{i \omega}{4} \int_{z}\left(\mathbf{u}_{m}^{\prime} \cdot \mathbf{t}_{-n}^{\prime}-\mathbf{u}_{-n}^{\prime} \cdot \mathbf{t}_{m}^{\prime}\right) d z=0 \text { if } m \neq n
$$

The above relation indeed represents a slightly modified version of the real biorthogonality relation of Auld [8]. Unlike Auld's complex orthogonality relation, the real relation remains valid for non-propagating modes and for lossy waveguides [14].

The above relation can also be written as $Q_{m n}=0$ for $m \neq-n$. As a consequence, one has $Q_{m,-n}+Q_{m, n}=0$ if $m \neq \pm n$. Let us define $I_{m n}$, from: $Q_{m,-n}+Q_{m, n}=\omega I_{m n} / 2$. Using the relation for the transverse isotropic case between forward and opposite-going modes (Appendix A), one has:

$$
I_{m n}=i \int_{z}\left(u_{m_{z}} \sigma_{n_{z^{\prime}}}-u_{n_{x^{\prime}}} \sigma_{m_{x^{\prime} x^{\prime}}}-u_{n_{y^{\prime}}} \sigma_{m_{y^{\prime} x^{\prime}}}\right) d z=0 \text { if } m \neq \pm n
$$

For Lamb modes $\left(u_{y^{\prime}}=0\right)$, the relation (75) corresponds to Fraser's relation [41]. For SH modes $\left(u_{x^{\prime}}=u_{z}=0\right)$, it corresponds to the well-known orthogonality relation: $\int u_{n, \gamma^{\prime}} u_{m,} d z=0$ if $m \neq \pm n$ (because $\sigma_{m \nu^{\prime} x^{\prime}}=i k\left(C_{11}-\right.$ $\left.\left.C_{12}\right) u_{m_{v^{\prime}}} / 2\right)$. These orthogonality relations for Lamb and SH modes are indeed the fundamental relations used in Achenbach's work [9].

As a consequence, the procedure followed with the SAFE method in this paper is in fact quite analogous to modal analytical approaches based on Auld's or Fraser's relations. This further justifies the direct link existing between numerical and analytical solutions described in Sec. 3.5.

\section{Example}

In this section, the example of a bilayer viscoelastic waveguide is considered to illustrate the method. Each layer is homogeneous although this is not a limitation of the SAFE method.

\subsection{Test case description}

The bilayered plate is taken from the work of Simonetti [49]. The relevant material parameters are summarized in Table 1. Complex bulk wave velocities are defined from:

$$
\tilde{c}_{s, l}=\frac{c_{s}}{1+\mathrm{i} \frac{\alpha_{s, l}}{2 \pi}}
$$

The matrix of elasticity coefficients $\mathbf{C}$ is complex. As already explained, the solutions proposed in this paper remain valid in this case.

As shown in Ref. [49], this test case yields strong viscoelastic effects upon dispersion curves compared to the lossless case. Additionally, the viscoelastic excitabilities have already been calculated with a SAFE method in Ref. [14] for the 2D line source problem.

Figure 3 shows the dispersion curves for the phase velocity and the attenuation computed with the SAFE method of this paper, for three modes of interest: two Lamb modes denoted $M_{0}$ and $M_{1}$ and one SH mode. For both Lamb modes, the numerical dispersion curves coincide with the analytical results of Ref. [49]. For the SH mode, the curves are found to have a behavior analogous to the $M_{1}$ mode, with lower velocity and stronger attenuation. 


\begin{tabular}{ccccccc}
\hline & $c_{s}(\mathrm{~m} / \mathrm{s})$ & $c_{l}(\mathrm{~m} / \mathrm{s})$ & $\rho\left(\mathrm{kg} / \mathrm{m}^{3}\right)$ & $\alpha_{s}(\mathrm{~Np} / \lambda)$ & $\alpha_{l}(\mathrm{~Np} / \lambda)$ & thickness $(\mathrm{mm})$ \\
\hline \hline Layer 1 (elastic) & 900 & 1700 & 1250 & 1 & 1 & 9 \\
Layer 2 (metallic) & 3260 & 5960 & 7930 & 0 & 0 & 8 \\
\hline
\end{tabular}

Table 1: Characteristics of the bilayered plate

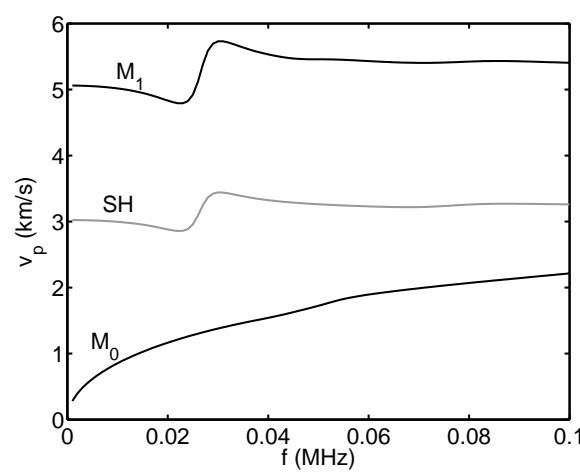

(a)

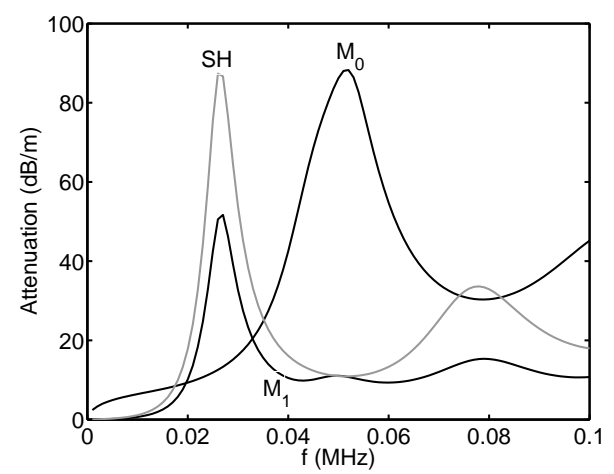

(b)

Figure 3: Dispersion curves in terms of frequency for: (a) phase velocity, (b) attenuation. Black: Lamb modes (denoted $M_{0}$ and $M_{1}$ ), gray: SH mode.

\subsection{Source description}

The excitation is a uniform rectangular source of length $a=20 \mathrm{~mm}$ and width $b=40 \mathrm{~mm}$ positioned at the surface of Layer 1 and centered at $(x, y)=(0,0)$. The $p$ th coefficient of the space 2D Fourier transform of the source profile has been given by Eq. (72), where the integral can be evaluated numerically by a trapezoidal method. However, such an expression is not suitable due to round-off errors that prevent convergence as the number of Fourier coefficients retained in the series is increased. These round-off errors are due to the fact that the integrand in Eq. (72) does not tend to zero as $p$ increases.

Instead, we can use a double integral analogous to Eq. (54) for calculating the surface source term. As shown in Appendix $\mathrm{B}$, the function $\tilde{f}_{p}(k)$ is also equal to the Hankel transform of order $p$ of the $p$ th coefficient of the Fourier series of $f(r, \theta)$ :

$$
\tilde{f}_{p}(k)=\frac{1}{2 \pi} \int_{0}^{\infty} \int_{0}^{2 \pi} f(r, \theta) e^{-i p \theta} d \theta J_{p}(k r) r d r
$$

In our example, $f(r, \theta)$ is equal to one inside the rectangular region delimited by $|x| \leqslant a / 2$ and $|y| \leqslant b / 2$, and is equal to zero outside. As a consequence, the integration on $\theta$ can be performed analytically, with integration bounds depending on $r$ (several regions must be distinguished). Assuming $a \leqslant b$, cumbersome calculations yield the following result:

$$
\tilde{f}_{p}(k)=\left\{\begin{array}{cc}
\int_{0}^{r_{s}} J_{0}(k r) r d r-\frac{2}{\pi} \int_{\frac{a}{2}}^{r_{s}} \theta_{a}(r) J_{0}(k r) r d r-\frac{2}{\pi} \int_{\frac{b}{2}}^{r_{s}} \theta_{b}(r) J_{0}(k r) r d r & \text { for } p=0 \\
-\frac{2}{p \pi} \int_{\frac{a}{2}}^{r_{s}} \sin \left(p \theta_{a}(r)\right) J_{p}(k r) r d r-\frac{2 i^{p}}{p \pi} \int_{\frac{b}{2}}^{r_{s}} \sin \left(p \theta_{b}(r)\right) J_{p}(k r) r d r & \text { for } p \neq 0 \text { even } \\
0 & \text { for } p \text { odd }
\end{array}\right.
$$

where $r_{s}=\sqrt{a^{2}+b^{2}} / 2, \theta_{a}(r)=\arccos (a / 2 r)$ and $\theta_{b}(r)=\arccos (b / 2 r)$. For a given $r$, each integrand of the above expression vanishes as $|p| \rightarrow \infty$ thanks to Bessel function properties. From a computation point of view, this ensures the convergence of series as the number of coefficients $p$ increases in the expansion.

\subsection{Reference method ( $2 D$ convolution)}

In the point source solution given by Eq. (59), the whole matrix inside the parentheses of the left-hand side can be identified as the Green's matrix, denoted as $\mathbf{H}$ in the following. By definition, the response displacement to a force 
arbitrarily distributed in the $(x, y)$ plane is given by the two-dimensional convolution product between the Green's matrix and that force.

The convolution must be written with respect to Cartesian coordinates and tensors must be written with respect to the Cartesian basis (convolution theory in polar coordinates can be found in Ref. [47] but tools are hardly available). The response in the Cartesian basis is hence calculated as follows:

$$
\left\{\begin{array}{l}
\mathbf{U}_{x} \\
\mathbf{U}_{y} \\
\mathbf{U}_{z}
\end{array}\right\}=\left(\mathbf{R}_{\theta}^{T} \mathbf{H}\right) * *\left\{\begin{array}{l}
\mathbf{F}_{x} \\
\mathbf{F}_{y} \\
\mathbf{F}_{z}
\end{array}\right\}
$$

where the 2D convolution product of arbitrary functions $f$ and $g$ is defined as:

$$
f(x, y) * * g(x, y)=\int_{-\infty}^{+\infty} \int_{-\infty}^{+\infty} f(x-\xi, y-\eta) g(\xi, \eta) d \xi d \eta
$$

The response in the cylindrical basis is obtained by left multiplying the response (79) by the matrix $\mathbf{R}_{\theta}$.

Since analytical calculations are not achievable, the response is approximated thanks to a discrete two-dimensional convolution. A rectangular $(x, y)$ grid of 512 by 512 points is defined. The Green's solution corresponding to a given displacement dof generated by a point force in a given direction is evaluated from Eq. (59) at each point of the rectangular grid. This Green's solution is projected onto the Cartesian basis, yielding a first matrix grid. The excitation is discretized on the same grid, yielding a second matrix grid. Then, the Cartesian displacement response is obtained from the discrete two-dimensional convolution between both grids. This response must be finally projected on the cylindrical basis to be compared with the solution (42).

Owing to the validation of Eq. (59) with the analytical solution presented in Sec. 4.3, results obtained from the convolution method can be considered as reference solutions. Yet the two-dimensional convolution method may be time-consuming (depending on the typical wavelengths to be discretized, on the source size...). With such a method, calculations are necessarily performed on the whole grid, even if attention is restricted to some points only.

Conversely, the solution given by Eq. (42) is particularly interesting from a computational point of view as it allows to perform single point calculations.

\subsection{Comparison between modal and convolution solutions}

Figure 4 shows the displacement fields $u_{z}$ and $u_{\theta}$ at $50 \mathrm{kHz}$ for an applied stress oriented normal to the plane ( $z$ direction). In this case, $\mathbf{F}_{x}=\mathbf{F}_{y}=\mathbf{0}$ : according to Eq. (42), no SH waves occur and only Lamb modes are present.

In the modal expansions, 10 positive-going modes and 10 Fourier coefficients $(|p| \leq 10)$ are retained. Numerical tests have shown that this truncation is sufficient to ensure convergence of results with good accuracy. As outlined in Sec. 3.2, one recalls that results calculated with the modal method are valid outside the source region (i.e. for $r>r_{s}$ ). This restriction also applies with the convolution method because the Green's solution (59) is singular at $r=0$. The region inside the source $\left(r \leq r_{s}\right)$ is hence not displayed.

As shown by Fig. 4, results computed with the modal and the convolution methods are in quite good agreement in the far field as well as near the source. The $z$ displacement exhibits a wide-angle radiation pattern (Fig. 4a), as opposed to its $\theta$ counterpart (Fig. 4c). Note that $u_{\theta}$ is not equal to zero, unlike for a normal point source, because the rectangular source generates $p \neq 0$ coefficients. It can be observed that the radiation pattern for $u_{\theta}$ mainly occurs for $p=2$. In fact, the coefficient $\mathbf{G}_{\theta z}^{(m, p)}$ always vanishes for $p=0$ (see Eq. (43)), while $\tilde{f}_{p}$ is zero if $p$ is odd owing to Eq. (78) ( $p=1$ also vanishes).

Figure 5 shows the displacement fields $u_{r}$ and $u_{\theta}$ at $50 \mathrm{kHz}$ for an applied stress oriented along the $x$ direction. Results obtained with the modal method again coincide with those of the convolution method. For an excitation along $x$, the examination of Eq. (42) tells us that both Lamb and SH waves are generated in the near-field but Lamb waves are predominant in the far field of $u_{r}$, while SH waves dominate the far-field of $u_{\theta}$. This can be clearly observed in Figs. 5a and 5c. Lamb waves are preferentially emitted in the $x$ direction (Fig 5a), while the SH wave (of longer wavelength) is rather emitted along $y$ (Fig 5c).

The results of modal and convolution methods have been compared for every other possible combination of displacement and source directions, with very good agreement (results not shown for conciseness). This further validates the theoretical developments presented in this paper. 


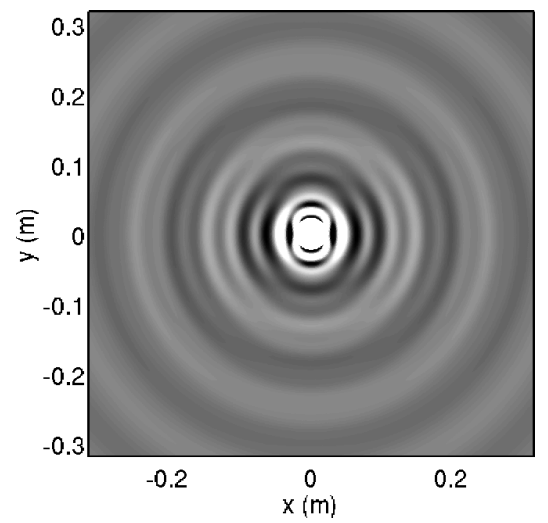

(a)

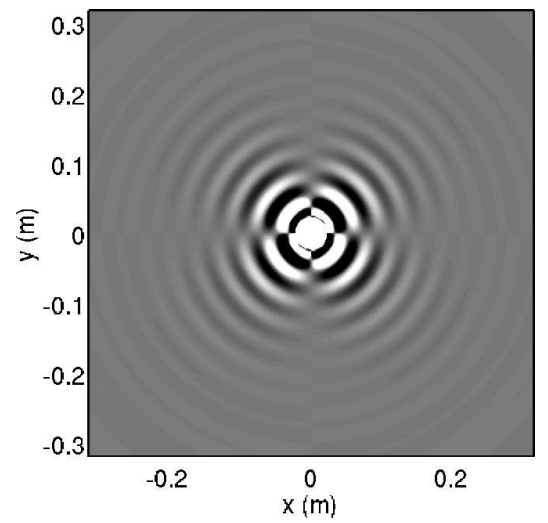

(c)

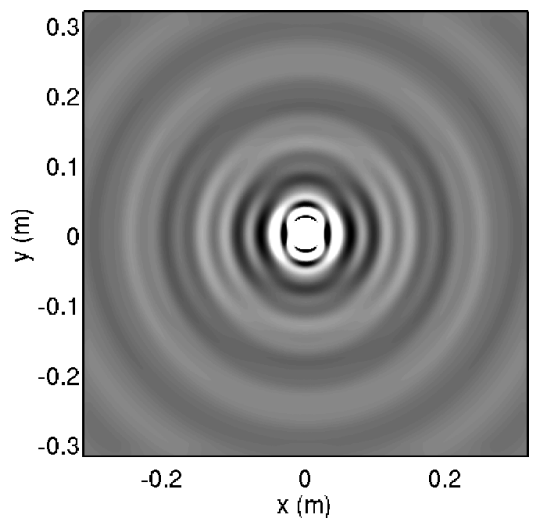

(b)

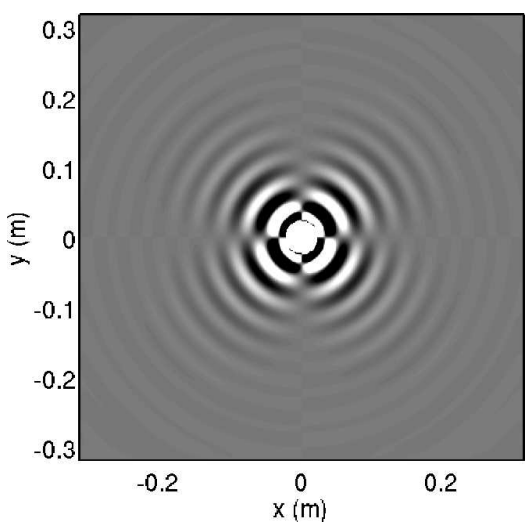

(d)

Figure 4: Response at $50 \mathrm{kHz}$ for an excitation normal to the plane ( $z$ direction) and evenly distributed on a rectangular surface 20x40mm, calculated for: (a) $u_{z}$ from modal expansion (i.e. Eq. (42)), (b) $u_{z}$ from convolution, (c) $u_{\theta}$ from modal method, (d) $u_{\theta}$ from convolution. The response is shown at the surface of Layer 1, where the excitation is applied. 


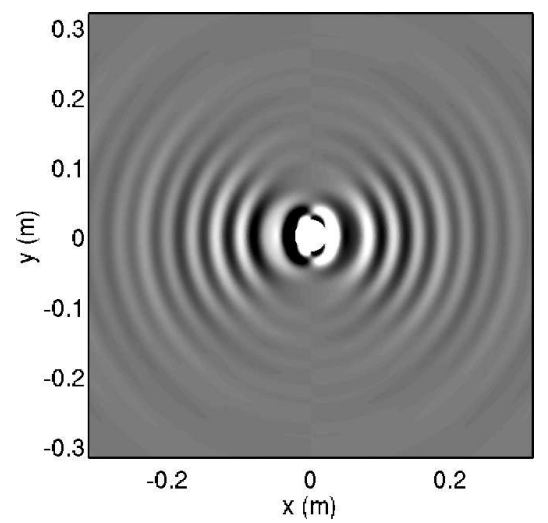

(a)

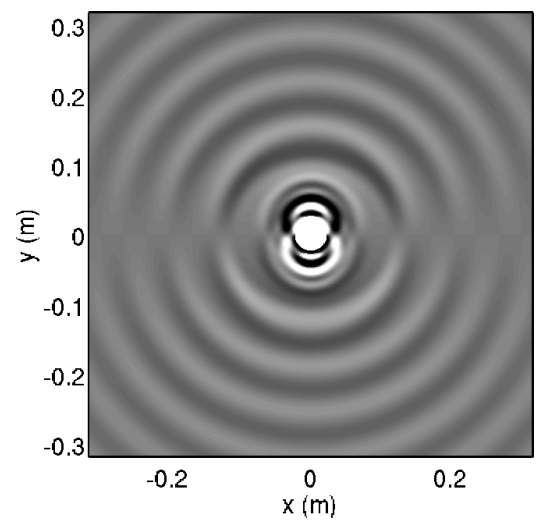

(c)

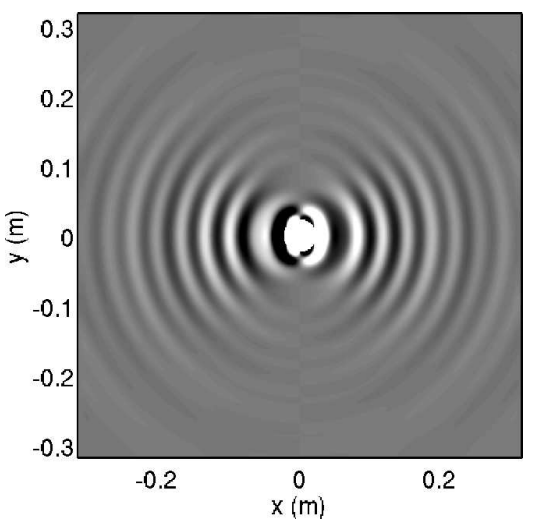

(b)

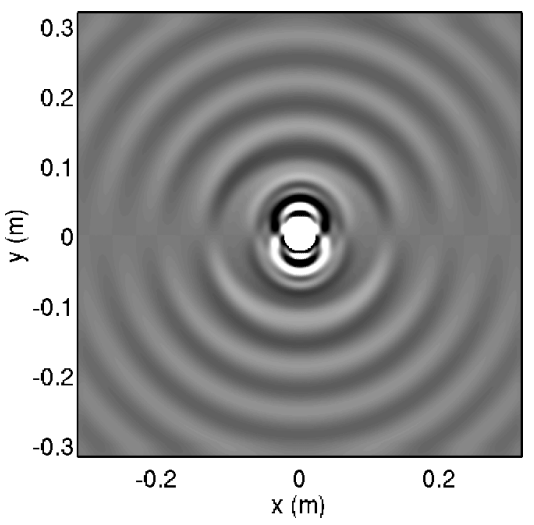

(d)

Figure 5: Response at 50kHz for an excitation tangent to the plane ( $x$ direction) and evenly distributed on a rectangular surface 20x40mm, calculated for: (a) $u_{r}$ from modal method, (b) $u_{r}$ from convolution, (c) $u_{\theta}$ from modal expansion, (d) $u_{\theta}$ from convolution. The response is shown at the surface of Layer 1, where the excitation is applied. 


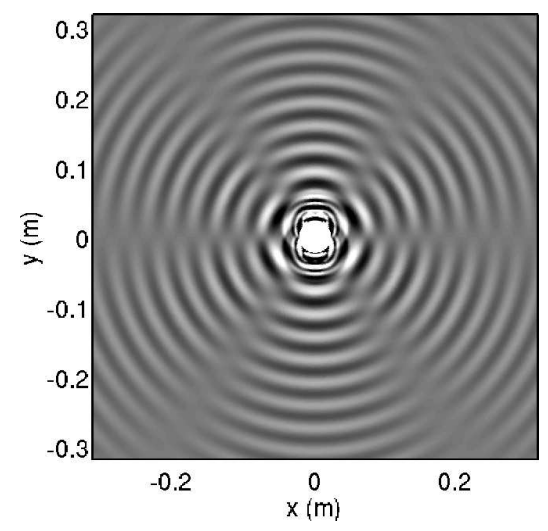

Figure 6: Same caption as Fig. 5c but for 100kHz.

In order to illustrate the change of radiation pattern with frequency, Fig. 6 gives the displacement field $u_{\theta}$ computed with the modal approach at $100 \mathrm{kHz}$ (excitation along $x$ ). The number of wave modes retained in the expansion is equal to 20 . As can be observed, the directivity pattern has strongly evolved compared to Fig. 5c.

\subsection{Time response}

In this section, the applied stress is oriented along the $x$ direction. The time excitation is a Hanning-windowed 5 cycles sinusoidal toneburst centered at $50 \mathrm{kHz}$. Computations are performed from the modal approach for 100 frequencies evenly spaced up to $100 \mathrm{kHz}$ and the inverse Fourier transform is processed.

Figure 7 gives the time response for $u_{r}$ and $u_{\theta}$ in the direction $\theta=\pi / 4$ at two distances, $r=45 \mathrm{~mm}$ and $r=$ $900 \mathrm{~mm}$, calculated from the exact solution (42) and from the far field approximation (47) (also given by Eq. (69) for a rectangular source). Note that the convolution method would yield cumbersome calculations here because the computation of the complete field in the $(x, y)$ plane would be required at each frequency step.

At $r=45 \mathrm{~mm}$, it can be observed that the far field approximation fails. Of course, the error committed by the far-field approximation would further increase closer to the source. Note that the error appears to be greater for $u_{\theta}$ (Fig. 7b), for which the SH wave mainly contributes as already explained in Sec. 5.4.

At $r=900 \mathrm{~mm}$, the far field solution yields accurate results. Time signals are superimposed with the exact solutions. For $u_{r}$, the main contribution is given by Lamb modes and two wave packets can be distinguished (Fig. 7c). The fastest packet corresponds to the $M_{1}$ mode, the slowest one to the $M_{0}$ mode. For $u_{\theta}$, the response is dominated by the SH wave. The time of flight of the SH mode is found to lie between those of $M_{0}$ and $M_{1}$ modes (compare Figs. $7 \mathrm{~d}$ with $7 \mathrm{c}$ ), as expected from the dispersion curves in Fig. 3.

\section{Conclusion}

In this paper, a 3D modal solution for Lamb and SH waves excited by sources of arbitrary shape has been derived under the assumption of transverse isotropy about the thickness direction. This solution is valid for elastic or viscoelastic media and in the far-field as well as in the near-field, including non-propagating modes (evanescent or inhomogeneous). It is written in terms of Hankel functions of arbitrary orders thanks to a proper application of inverse transforms and Cauchy residue calculus. The proposed solution has been simplified in the far field. Theoretical developments have been conducted on the basis of a SAFE method, which allows to readily handle heterogeneous materials having depth-varying properties (multilayered, piecewise, or continuously varying as in the case of functionally graded materials) with fast computations (the SAFE modal problem being one-dimensional).

The link with a fully analytical approach has also been established. It has been shown that the procedure followed with the SAFE method is in fact quite analogous to modal analytical approaches based on Auld's or Fraser's relations.

The solution proposed in this paper has been successfully compared to various literature results obtained in special cases. The general expression degenerate to Achenbach's point source solution. Useful formula have been presented 


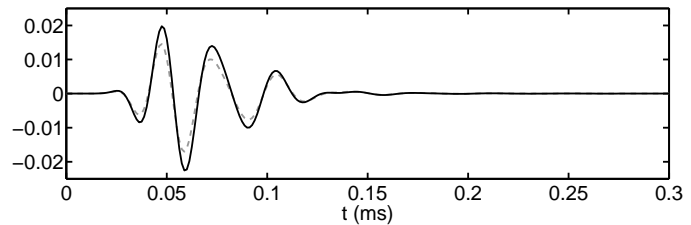

(a)

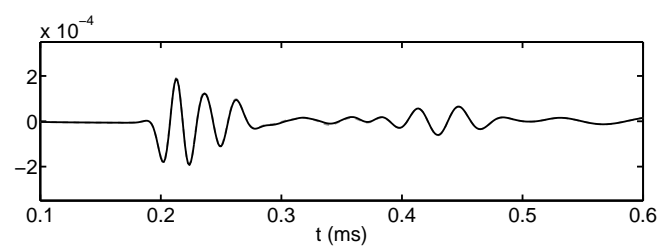

(c)

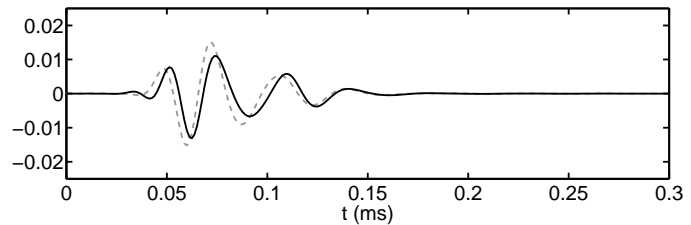

(b)

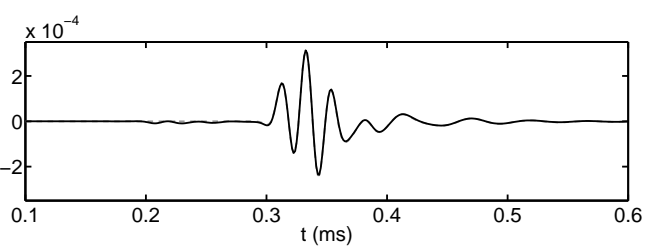

(d)

Figure 7: Time response calculated at $\theta=\pi / 4$ for: (a) $u_{r}$ at $r=45 \mathrm{~mm}$, (b) $u_{\theta}$ at $r=45 \mathrm{~mm}$, (c) $u_{r}$ at $r=900 \mathrm{~mm}$, (d) $u_{\theta}$ at $r=900 \mathrm{~mm}$.. Black continuous lines: exact solution given by Eq. (42), gray dashed lines: far-field solution given by Eq. (47) (black lines are superimposed on gray lines in Figs. (c) and (d)). The excitation is oriented in the $x$ direction and uniformly distributed on a rectangular surface 20x40 mm. Excitation and response are both at the surface of Layer 1.

to calculate point source excitabilities from lines sources. These excitabilities remain valid for non-propagating modes and viscoelastic materials and the associated propagators can account for the near field effect.

Finally, the example of a bilayer waveguide excited by a rectangular source has been considered. The theoretical developments have been checked by comparing numerical results with those obtained by the two-dimensional convolution of the point source solution.

\section{Appendix A. Calculation of opposite-going modes from forward-going ones}

The following relation can be obtained between pairs of forward and opposite-going modes, denoted $\left(k_{m}, \mathbf{u}_{m}^{\prime}\right)$ and $\left(-k_{m}, \mathbf{u}_{-m}^{\prime}\right)$ respectively:

$$
u_{-m_{x^{\prime}}}=u_{m_{x^{\prime}}}, \quad u_{-m_{y^{\prime}}}=u_{m_{y^{\prime}}}, \quad u_{-m_{z}}=-u_{m_{z}}
$$

These identities can be checked from Eqs. (7) and (8) by accounting for the particular expression of $\mathbf{C}$ in the transverse isotropic case. Hence with the SAFE method, an opposite-going eigenvector can be deduced from the forward one by:

$$
\mathbf{U}_{-m}^{\prime}=\left\{\begin{array}{l}
\mathbf{U}_{-m_{x^{\prime}}} \\
\mathbf{U}_{-m_{y^{\prime}}} \\
\mathbf{U}_{-m_{z}}
\end{array}\right\}=\left\{\begin{array}{c}
\mathbf{U}_{m_{x^{\prime}}} \\
\mathbf{U}_{m_{y^{\prime}}} \\
-\mathbf{U}_{m_{z}}
\end{array}\right\}
$$

For the stress tensor, the following identities can be shown:

$$
\sigma_{-m_{x^{\prime} x^{\prime}}}=-\sigma_{m_{x^{\prime} x^{\prime}}}, \quad \sigma_{-m_{y^{\prime} x^{\prime}}}=-\sigma_{m_{y^{\prime} x^{\prime}}}, \quad \sigma_{-m_{z x^{\prime}}}=\sigma_{m_{z x^{\prime}}}
$$

which yields for SAFE modal forces:

$$
\mathbf{F}_{-m}^{\prime}=\left\{\begin{array}{l}
\mathbf{F}_{-m_{x^{\prime}}} \\
\mathbf{F}_{-m_{y^{\prime}}} \\
\mathbf{F}_{-m_{z}}
\end{array}\right\}=\left\{\begin{array}{c}
-\mathbf{F}_{m_{x^{\prime}}} \\
-\mathbf{F}_{m_{y^{\prime}}} \\
\mathbf{F}_{m_{z}}
\end{array}\right\}
$$




\section{Appendix B. Link between the 2D Fourier transform and the Hankel transform}

Let us start from Eq. (2), the 2D Fourier transform of an arbitrary function $f(x, y)$. The application of a polar change of variable yields:

$$
\tilde{f}(k, \phi)=\int_{0}^{2 \pi} \int_{0}^{\infty} f(r, \theta) e^{-i k r \cos (\theta-\phi)} r d r d \theta
$$

The function $f(r, \theta)$ is expanded as a Fourier series:

$$
f(r, \theta)=\sum_{p=-\infty}^{+\infty} f_{p}(r) e^{i p \theta}
$$

where $f_{p}(r)=\frac{1}{2 \pi} \int_{0}^{2 \pi} f(r, \theta) e^{-i p \theta} d \theta$. Using this expansion into Eq. (B.1) and the property (36), one obtains:

$$
\tilde{f}(k, \phi)=2 \pi \sum_{p=-\infty}^{+\infty} i^{p} e^{i p \phi} \int_{0}^{\infty} f_{p}(r) J_{p}(-k r) r d r
$$

From the property $J_{p}(-k r)=(-1)^{p} J_{p}(k r)$, the 2D Fourier transform of $f(x, y)$ in polar variables is finally:

$$
\tilde{f}(k, \phi)=2 \pi \sum_{p=-\infty}^{+\infty} i^{-p} \tilde{f}_{p}(k) e^{i p \phi}
$$

where $\tilde{f}_{p}(k)=\int_{0}^{\infty} f_{p}(r) J_{p}(k r) r d r$ denotes the Hankel transform of order $p$ of $f_{p}(r)$. More details on Fourier transforms in polar coordinates can be found in [47].

\section{Appendix C. Application of Cauchy residue theorem to inverse Hankel transforms}

For $k r \geq 0$, the following identity holds:

$$
J_{q}(k r)=\frac{1}{2} H_{q}^{(1)}(k r)+\frac{1}{2} H_{q}^{(2)}(k r)=\left\{\begin{array}{l}
\frac{1}{2} H_{q}^{(1)}(k r)-\frac{1}{2} H_{q}^{(1)}(-k r) \text { for } q \text { even } \\
\frac{1}{2} H_{q}^{(1)}(k r)+\frac{1}{2} H_{q}^{(1)}(-k r) \text { for } q \text { odd }
\end{array}\right.
$$

Besides, the Hankel transform of order $q$ of a function $f(r)$, denoted $\tilde{f}_{q}(k)$, is always an even function when $q$ is even, and an odd function for $q$ odd. As a consequence, the inverse Hankel transform of order $q$ can be rewritten as:

$$
f(r)=\frac{1}{2} \int_{-\infty}^{+\infty} \tilde{f}_{q}(k) H_{q}^{(1)}(k r) k d k
$$

for any integer $q$. The inverse Hankel transform of order $q$ given by Eq. (37) can hence be rewritten as follows:

$$
f(r)=\frac{1}{2} \int_{-\infty}^{+\infty} \frac{\tilde{g}_{q}(k)}{k-k_{m}} H_{q}^{(1)}(k r) k d k
$$

The above form is well suited for the application of the Cauchy residue theorem, which yields the result given by Eq. (38).

\section{Appendix D. Calculation of $\mathbf{G}_{r r}^{(m, p)}$}

To calculate $\mathbf{G}_{r r}^{(m, p)}$, let us write the radial displacement vector $\mathbf{U}_{r}$ excited by the radial excitation vector $\tilde{\mathbf{F}}_{r}$ (we set $\tilde{\mathbf{F}}_{\theta}=\tilde{\mathbf{F}}_{z}=\mathbf{0}$ ). From Eqs. (35) and (31), one has:

$$
\begin{array}{r}
\mathbf{U}_{r}=-\frac{i}{2 \pi} \sum_{m=1}^{2 M} \sum_{p=-\infty}^{+\infty} i^{-p} e^{i p \theta} \int_{0}^{+\infty} \int_{0}^{2 \pi}\left(\mathbf{E}_{m_{x^{\prime} x^{\prime}}} \cos ^{2} \varphi\right. \\
\left.+\mathbf{E}_{m_{y^{\prime} y^{\prime}}} \sin ^{2} \varphi\right) e^{i k r \cos \varphi+i p \varphi} d \varphi \frac{\tilde{\mathbf{F}}_{p_{r}}}{k-k_{m}} k d k
\end{array}
$$


From the identities $\cos ^{2} \varphi=\left(2+e^{2 i \varphi}+e^{-2 i \varphi}\right) / 4$ and $\sin ^{2} \varphi=\left(2-e^{2 i \varphi}-e^{-2 i \varphi}\right) / 4$, the above expression becomes thanks to Eq. (36):

$$
\begin{array}{r}
\mathbf{U}_{r}=-\frac{i}{4} \sum_{m=1}^{2 M} \sum_{p=-\infty}^{+\infty} e^{i p \theta} \int_{0}^{+\infty}\left(\mathbf{E}_{m_{x^{\prime} x^{\prime}}}\left(2 J_{p}(k r)-J_{p+2}(k r)-J_{p-2}(k r)\right)\right. \\
\left.+\mathbf{E}_{m_{y^{\prime} y^{\prime}}}\left(2 J_{p}(k r)+J_{p+2}(k r)+J_{p-2}(k r)\right)\right) \frac{\tilde{\mathbf{F}}_{p_{r}}}{k-k_{m}} k d k
\end{array}
$$

Then applying the Cauchy residue theorem (Appendix C), one gets:

$$
\begin{array}{r}
\mathbf{U}_{r}=\frac{1}{4} \sum_{m=1}^{M} \sum_{p=-\infty}^{+\infty}\left(2\left(\mathbf{E}_{m_{x^{\prime} x^{\prime}}}+\mathbf{E}_{m_{y^{\prime} y^{\prime}}}\right) H_{p}^{(1)}\left(k_{m} r\right)\right. \\
\left.-\left(\mathbf{E}_{m_{x^{\prime} x^{\prime}}}-\mathbf{E}_{m_{y^{\prime} y^{\prime}}}\right)\left(H_{p+2}^{(1)}\left(k_{m} r\right)+H_{p-2}^{(1)}\left(k_{m} r\right)\right)\right) \tilde{\mathbf{F}}_{p_{r}}\left(k_{m}\right) \pi k_{m} e^{i p \theta}
\end{array}
$$

In order to explicit far-field terms, the function $H_{p+2}^{(1)}\left(k_{m} r\right)$ and $H_{p-2}^{(1)}\left(k_{m} r\right)$ are rewritten as follows:

$$
\begin{array}{r}
H_{p-2}^{(1)}\left(k_{m} r\right)=\frac{2(p-1)}{k_{m} r}\left\{\frac{2 p}{k_{m} r} H_{p}^{(1)}\left(k_{m} r\right)-H_{p+1}^{(1)}\left(k_{m} r\right)\right\}-H_{p}^{(1)}\left(k_{m} r\right), \\
H_{p+2}^{(1)}\left(k_{m} r\right)=\frac{2(p+1)}{k_{m} r} H_{p+1}^{(1)}\left(k_{m} r\right)-H_{p}^{(1)}\left(k_{m} r\right)
\end{array}
$$

Finally, using Eq. (D.4) into (D.3), the following result is obtained:

$$
\begin{array}{r}
\mathbf{U}_{r}=\sum_{m=1}^{M} \sum_{p=-\infty}^{+\infty}\left\{\mathbf{E}_{m_{x^{\prime} x^{\prime}}} H_{p}^{(1)}\left(k_{m} r\right)\right. \\
-\left(\mathbf{E}_{m_{x^{\prime} x^{\prime}}}-\mathbf{E}_{m_{y^{\prime} y^{\prime}}}\left(\frac{H_{p+1}^{(1)}\left(k_{m} r\right)}{k_{m} r}+(p-1) p \frac{H_{p}^{(1)}\left(k_{m} r\right)}{\left(k_{m} r\right)^{2}}\right)\right\} \tilde{\mathbf{F}}_{p_{r}}\left(k_{m}\right) \pi k_{m} e^{i p \theta}
\end{array}
$$

Identifying Eq. (39) with (D.5) leads to the expression of $\mathbf{G}_{r r}^{(m, p)}$.

\section{References}

[1] P. D. Wilcox, M. J. S. Lowe, P. Cawley, Mode and transducer selection for long range Lamb wave inspection, Journal of Intelligent Material Systems and Structures 12 (2001) 553-565.

[2] I. A. Viktorov, Rayleigh and Lamb Waves, Plenum, New York, 1967, 154 p.

[3] J. D. Achenbach, Wave Propagation in Elastic Solids, North-Holland, Amsterdam, the Netherlands, 1973,425 p.

[4] W. A. Green, E. R. Green, Elastic waves in fibre composite laminates, Ultrasonics 38 (2000) 228-231.

[5] E. V. Glushkov, N. V. Glushkova, W. Seemann, O. V. Kvasha, Elastic wave excitation in a layer by piezoceramic patch actuators, Acoustical Physics 52 (2006) 398-407.

[6] S. Banerjee, C. B. Pol, Theoretical modeling of guided wave propagation in a sandwich plate subjected to transient surface excitations, International Journal of Solids and Structures 49 (2012) 3233-3241.

[7] C. B. Pol, S. Banerjee, Modeling and analysis of propagating guided wave modes in a laminated composite plate subject to transient surface excitations, Wave Motion 50 (2013) 964-978.

[8] B. A. Auld, Acoustic Fields and Waves in Solids, 2nd Edition, Vol. II, Krieger, Malabar, FL, 1990, 432 p.

[9] J. D. Achenbach, Reciprocity in elastodynamics, Cambridge University Press, Cambridge, UK, 2003,255 p.

[10] I. Nunez, R. K. Ing, C. Negreira, M. Fink, Transfer and green functions based on modal analysis for Lamb waves generation, Journal of the Acoustical Society of America 107 (2000) 2370-2378.

[11] P. D. Wilcox, Lamb wave inspection of large structures using permanently attached transducers, Ph.D. thesis, Imperial College of Science, Technology and Medecine, University of London (1998).

[12] P. Wilcox, M. Evans, O. Diligent, P. Cawley, Dispersion and excitability of guided acoustic waves in isotropic beams with arbitrary crosssection, in: D. O. Thompson, D. E. Chimenti (Eds.), Review of Progress in Quantitative NDE, Vol. 615, AIP Conference Proceedings, New York, 2002, pp. 203-210.

[13] A. Velichko, P. D. Wilcox, Modeling the excitation of guided waves in generally anisotropic multilayered media, Journal of the Acoustical Society of America 121 (2007) 60-69. 
[14] F. Treyssède, L. Laguerre, Numerical and analytical calculation of modal excitability for elastic wave generation in lossy waveguides, Journal of the Acoustical Society of America 133 (6) (2013) 3827-3837.

[15] N. Vasudevan, A. K. Mal, Response of an elastic plate to localized transient sources, Journal of Applied Mechanics 52 (2) (1985) $356-362$.

[16] R. L. Weaver, Y. H. Pao, Axisymmetric elastic-waves excited by a point-source in a plate, Journal of Applied Mechanics 49 (4) (1982) 821-836.

[17] F. Santosa, Y. H. Pao, Transient axially asymmetric response of an elastic plate, Wave Motion 11 (3) (1989) $271-295$.

[18] J. J. Ditri, Utilization of guided waves for the characterization of circumferential cracks in hollow cylinders, Journal of the Acoustical Society of America 96 (1994) 3769-3775.

[19] V. A. Babeshko, E. V. Glushkov, N. V. Glushkova, Energy vortices and backward fluxes in elastic wave-guides, Wave Motion 16 (3) (1992) 183-192.

[20] R. L. Weaver, W. Sachse, K. Y. Kim, Transient elastic waves in a transversely isotropic plate, Journal of Applied Mechanics 63 (2) (1996) $337-346$.

[21] A. Cheng, T. W. Murray, J. D. Achenbach, Simulation of laser-generated ultrasonic waves in layered plates, Journal of the Acoustical Society of America 110 (2001) 2902-2913.

[22] A. K. Mal, S.-S. Lih, Elastodynamic response of a unidirectional composite laminate to concentrated surface loads: part I, Journal of Applied Mechanics 59 (1992) 878-886.

[23] S.-S. Lih, A. K. Mal, Elastodynamic response of a unidirectional composite laminate to concentrated surface loads: part II, Journal of Applied Mechanics 59 (1992) 887-892.

[24] A. Mal, Elastic waves from localized sources in composite laminates, International Journal of Solids and Structures 39 (2002) $5481-5494$.

[25] J. D. Achenbach, Lamb waves as thickness vibrations superimposed on a membrane carrier wave, Journal of the Acoustical Society of America 103 (5) (1998) 2283-2286.

[26] J. D. Achenbach, Y. Xu, Use of elastodynamic reciprocity to analyze point-load generated axisymmetric waves in a plate, Wave Motion 30 (1) (1999) 57-67.

[27] P. Wilcox, Modeling the excitation of lamb and sh waves by point and line sources, Review of Progress in Quantitative Nondestructive Evaluation 23 (2004) 206-213.

[28] E. Moulin, S. Grondel, M. Baouahi, J. Assaad, Pseudo-3d modeling of a surface-bonded lamb wave source, Journal of the Acoustical Society of America 119 (5) (2006) 2575-2578.

[29] S. Guilbaud, B. Audoin, Measurement of the stiffness coefficients of a viscoelastic composite material with laser-generated and detected ultrasound, Journal of the Acoustical Society of America 105 (1999) 2226-2235.

[30] A. E. Martinez-Castro, R. Gallego, Three-dimensional green's function for time-harmonic dynamics in a viscoelastic layer, International Journal of Solids and Structures 44 (13) (2007) 4541-4558.

[31] L. Gavric, Computation of propagative waves in free rail using a finite element technique, Journal of Sound and Vibration 185 (1995) 531543.

[32] T. Hayashi, W.-J. Song, J. L. Rose, Guided wave dispersion curves for a bar with an arbitrary cross-section, a rod and rail example, Ultrasonics 41 (2003) 175-183.

[33] V. Damljanovic, R. L. Weaver, Propagating and evanescent elastic waves in cylindrical waveguides of arbitrary cross-section, Journal of the Acoustical Society of America 115 (2004) 1572-1581.

[34] I. Bartoli, A. Marzani, F. L. di Scalea, E. Viola, Modeling wave propagation in damped waveguides of arbitrary cross-section, Journal of Sound and Vibration 295 (2006) 685-707.

[35] P. W. Loveday, Simulation of piezoelectric excitation of guided waves using waveguide finite elements, IEEE Transactions on Ultrasonics, Ferroelectrics, and Frequency Control 55 (2008) 2038-2045.

[36] G. R. Liu, K. Y. Lam, Y. Ohyoshi, A technique for analyzing elastodynamic responses of anisotropic laminated plates to line loads, Composites Part B 28 (1997) 667-677.

[37] M. Veidt, T. Liu, S. Kitipornchai, Modelling of lamb waves in composite laminated plates excited by interdigital transducers, Ndt \& E International 35 (7) (2002) 437-447.

[38] M. J. S. Lowe, Plate waves for the ndt of diffusion bonded titanium, Ph.D. thesis, Mechanical Engineering Department, Imperial College London (1992).

[39] B. Pavlakovic, Leaky guided ultrasonic waves in ndt, Ph.D. thesis, Mechanical Engineering Department, Imperial College London (1998).

[40] H. Bai, J. Zhu, A. H. Shah, N. Popplewell, Three-dimensional steady state green function for a layered isotropic plate, Journal of Sound and Vibration 269 (1-2) (2004) 251-271.

[41] W. B. Fraser, Orthogonality relation for rayleigh-lamb modes of vibration of a plate, Journal of the Acoustical Society of America 59 (1) (1976) 215-216.

[42] A. Mahmoud, A. H. Shah, S. B. Dong, Transient response of transversely isotropic composite plates to a point source, Journal of Applied Mechanics 73 (2006) 338-341.

[43] B. Chapuis, N. Terrien, D. Royer, Excitation and focusing of lamb waves in a multilayered anisotropic plate, Journal of the Acoustical Society of America 127 (2010) 198-203.

[44] F. Tisseur, K. Meerbergen, The quadratic eigenvalue problem, SIAM Review 43 (2001) 235-286.

[45] S. Adhikari, M. I. Friswell, Eigenderivative analysis of asymmetric non-conservative systems, International Journal for Numerical Methods in Engineering 51 (2001) 709-733.

[46] F. Benmeddour, F. Treyssède, L. Laguerre, Numerical modeling of guided waves interaction with non-axisymmetric cracks in elastic cylinders, International Journal of Solids and Structures 48 (2011) 764-774.

[47] N. Baddour, Operational and convolution properties of two-dimensional Fourier transforms in polar coordinates, Journal of the Optical Society of America 26 (8) (2009) 1767-1777.

[48] J. D. Achenbach, Simplifications for the calculation of surface wave pulses generated by laser-irradiation, Journal of the Acoustical Society of America 116 (3) (2004) 1481-1487. 
[49] F. Simonetti, Sound propagation in lossless waveguides coated with attenuative materials, Ph.D. thesis, Imperial College of Science, Technology and Medecine, University of London (2003). 